\title{
MODELADO DE NICHO ECOLÓGICO DE LAS ESPECIES DEL GÉNERO Abies (Pinaceae) en México: Algunas implicaciones TAXONÓMICAS Y PARA LA CONSERVACIÓN
}

\author{
Norberto Martínez-Méndez ${ }^{1,3}$, Erika Aguirre-Planter ${ }^{2}$, Luis E. Eguiarte ${ }^{2}$ \\ y Juan Pablo Jaramillo-Correa ${ }^{2}$ \\ ${ }^{1}$ Laboratorio de Bioconservación y Manejo, Departamento de Zoología, \\ Escuela Nacional de Ciencias Biológicas del Instituto Politécnico Nacional, México, D.F., México \\ ${ }^{2}$ Departamento de Ecología Evolutiva, Instituto de Ecología, Universidad Nacional Autónoma de México, \\ México, D.F., México \\ ${ }^{3}$ Autor de correspondencia: nomartinezm@ipn.mx
}

\begin{abstract}
Resumen: En Mesoamérica y el norte de México, los oyameles o abetos (Abies, Pinaceae) presentan una distribución disyunta en áreas montañosas y templadas. En esta área se han reconocido entre seis y diez especies de Abies dependiendo de la propuesta taxonómica empleada; generalmente se considera que seis de éstas son endémicas a México y están incluidas en alguna categoría de riesgo. Dada esta incertidumbre taxonómica y la importancia económica del género (Abies es el cuarto recurso maderable de México), se necesita tener información adicional que contribuya a su manejo sustentable. En este estudio se obtuvo los modelos de nicho ecológico para ocho de las especies y dos variedades de Abies reconocidas en México (A. concolor, A. durangensis var. duragensis, A. durangensis var. coahuilensis, A. flincki, A. guatemalensis, A. hickelii, A. jaliscana, A. religiosa y A. vejari) a través del algoritmo de máxima entropía (Maxent) y utilizando variables bioclimáticas y topográficas provenientes de Worldclim, de Hydro $1 \mathrm{k}$ y datos depurados de presencia de diversas fuentes (CONABIO, GBIF, MEXU). Los resultados sugieren que A. concolor tiene el nicho ecológico más diferenciado con respecto a los demás abetos mexicanos. Asimismo, se observó que, con excepción de A. religiosa, el área de mayor idoneidad ambiental para todas las especies está fuera de la poligonal de algún área natural protegida ya sea federal o estatal, por lo que se proponen ampliar o crear nuevas áreas que protejan a estas especies.
\end{abstract}

Palabras clave: Abies, conservación, distribución geográfica, México, nicho ecológico.

\begin{abstract}
In Mexico and the northern part of Central America, firs (Abies, Pinaceae) are distributed disjunctly in temperate mountain zones. Depending on the taxonomic proposal used, six to ten species of Abies have been recognized for this area, six of which are generally considered endemic to Mexico and included in different risk categories. Given such taxonomic uncertainty and the economic importance of the genus (e.g. Abies is the fourth timber resource of Mexico), more information is needed for the sustainable management of all these taxa. Herein, ecological niche models were determined for eight species and two varieties of Abies currently recognized in Mexico (A. concolor, A. durangensis var. duragensis, A. durangensis var. coahuilensis, A. flincki, A. guatemalensis, A. hickelii, A. jaliscana, A. religiosa, and A. vejari) by using the Maximum entropy algorithm available in Maxent. The bioclimatic and topographyc layers included in the models were gathered from Worldclim and Hydrolk, while the occurrence data were obtained from various "cleaned" databases (CONABIO, GBIF, MEXU). The results suggest that A. concolor has the more distinct ecological niche among all Mesoamerican firs. Furthermore, and with the exception of A. religiosa, the high suitability areas for all fir taxa lay outside any federal or state protected area, which highlights the need of expanding or creating new protected areas for these species.
\end{abstract}

Key words: Abies, conservation, ecological niche, geographic distribution, Mexico.

$\mathbf{E}_{t}^{1}$ género Abies Mill. (oyameles, Pinaceae) está constituido por especies de afinidad boreal y templada distribuidas en Norteamérica y Eurasia y por algunos taxa en las zonas montañosas de Mesoamérica (Farjon, 1990, 2010; Farjon y Filer, 2013). El origen del género se ha datado en- tre el periodo Cretácico tardío y el Eoceno temprano y probablemente ocurrió en Norteamérica (Xiang et al., 2015); según el registro fósil su llegada a Mesoamerica no se dio hasta el Plioceno (Graham, 1999), aunque estudios moleculares han propuesto un arribo y diversificación más tem- 
pranos (Aguirre-Planter et al., 2012). En México, el género está representado por seis a diez especies, dependiendo de la propuesta taxonómica que se consulte. Todas ellas han sido delimitadas a partir de caracteres morfológicos y tienen una distribución disyunta y restringida a las partes más altas y húmedas de las principales cadenas montañosas del país (Martínez, 1948; Liu, 1971; Farjon, 1990; Debreczy y Rácz, 1995). Esta clasificación es aun tema de gran debate; de hecho, parece no haber un consenso sobre el concepto de especie que se debe utilizar para separar los taxa (p.ej. Vázquez-García et al., 2014), mientras que los ensayos para evaluar si los caracteres morfológicos utilizados en su identificación tienen un componente heredable o son fruto de plasticidad fenotípica no existen. Asimismo, los datos genéticos sugieren que existen menos especies de las que la morfología nos indica (p.ej. Aguirre-Planter et al., 2012), mientras que el componente ecológico aún no se ha integrado de manera formal en la separación de especies. En este sentido, dada la información filogenética disponible, que indica que las especies mexicanas tienen un mismo ancestro común (Aguirre-Planter et al., 2000, 2012; Semerikova y Semerikov 2014; Xiang et al., 2015) y cuyos taxa más divergentes son Abies concolor (Gordon \& Glend.) Hildebr. y Abies flinckii Rushforth (Aguirre-Planter et al., 2012), se esperaría que esta divergencia evolutiva se reflejara también a nivel ecológico. Además, dada la poca diferenciación genética (Aguirre-Planter et al., 2000; Jaramillo-Correa et al., 2008) y morfológica (Strandby et al., 2009) de los demás taxa, se esperaría que estos tuvieran nichos ecológicos muy similares y que se traslaparan, al menos, parcialmente. Sin embargo, los estudios sobre modelado de nicho ecológico de coníferas, y en especial de abetos en México, son pocos, por ejemplo Sáenz-Romero et al. (2012) modelaron el nicho ecológico de Abies religiosa (Kunth) Schltdl. \& Cham. en relación con su importancia como refugio de la mariposa monarca y las afectaciones ante distintos escenarios de cambio climático. Los otros estudios existentes se enfocan en utilizar el modelado de nicho ecológico como herramienta auxiliar para explicar algunos patrones filogeográficos (Gugger et al., 2011; Moreno-Letelier et al., 2013), pero ninguno de los estudios mencionados ha sido enfocado explícitamente como una método auxiliar en la delimitación de especies.

Por otra parte, aunque sus bosques abarcan alrededor de $143,579.28$ ha que es un área relativamente pequeña del territorio nacional, los oyameles constituyen el cuarto recurso maderable de México (SEMARNAT, 2007), a lo que hay sumar los servicios ambientales que proporcionan, como la captación y filtración de agua, la generación de oxígeno, la captación de carbono, la retención del suelo y el refugio que proporcionan a especies clave, como la mariposa monarca (Gómez-Gómez, 2004; Cayuela, 2006; Cuevas-Guzmán et al., 2011; Sáenz-Romero et al., 2012; Martínez-Arévalo, 2013; Pineda-López et al., 2013). Sin embargo, como con- secuencia directa del mal manejo y de la tala clandestina (Alvarado et al., 1991), seis de las especies de Abies reconocidas para México se encuentran en alguna de las categorías de riesgo (SEMARNAT, 2010 y IUCN, 2011) y son parte del "Listado preliminar de especies incluidas en el sistema de información del Inventario Nacional Forestal y de Suelos (INFyS)" de la CONAFOR. Este último es una de las principales fuentes de información con las que cuenta el gobierno mexicano para tomar decisiones e implementar políticas públicas de conservación y manejo sustentable de los recursos forestales (Sánchez-Velásquez, 2002; CONAFOR, 2012). Ahora bien, si se tienen en cuenta todas las incertidumbres taxonómicas arriba mencionadas, la conclusión obvia es que la implementación de estas políticas de conservación se verán limitadas, ya que es difícil establecer programas para especies que no están delimitadas unánimemente por la comunidad científica. Con el ánimo de contribuir a este debate, en este estudio se utilizaron modelos de nicho ecológico que podrán ser empleados posteriormente para ayudar a resolver algunos problemas taxonómicos para el género Abies y entender los procesos evolutivos que han generado su diversidad. A su vez, también se buscó identificar zonas en donde los requerimientos ambientales para su desarrollo sean los adecuados y que podrían ser integradas en los programas de conservación existentes.

\section{Materiales y Métodos}

Consideraciones taxonómicas. Para la asignación de las poblaciones estudiadas a las distintas especies de Abies mesoamericanos seguimos a Aguirre-Planter et al. (2012), que a su vez se apoyó en Liu (1971) y Farjon y Rushforth (1989), quienes consideran que en México existen ocho especies de Abies, seis de las cuales son endémicas al país. Siguiendo este criterio, algunos datos de presencia se reasignaron según los parámetros marcados por estos autores. Por ejemplo, apoyados por datos morfológicos y genéticos se consideró a Abies mexicana Martínez como sinónimo de Abies vejarii Martínez, por lo que los datos de ocurrencia de ambos taxa fueron fusionados. De igual manera, los datos de Abies colimensis Rushforth \& Narave se asignaron a los de A. religiosa por ser una especie sinónima de esta última (Farjon, 2013). En el caso de los demás abetos del occidente de México, la situación es un poco más complicada ya que de acuerdo con Rushfort (1989) los registros de Abies guatemalensis Rehder var. jaliscana Martínez y de Abies religiosa var. emarginata Loock \& Martínez (Martínez, 1948) estarían en sinonimia con A. flinckii (Rushforth, 1989). Esta última especie, aunque no es reconocida por algunas autoridades, ha mostrado una diferencia genética significativa con respecto a sus congéneres (Aguirre-Planter $e t$ al., 2000, 2012; Jaramillo-Correa et al., 2008), por lo que es de interés ecológico y evolutivo ver si ésta se refleja al nivel de su nicho ecológico. Sin embargo, en un trabajo reciente 
Vázquez-García et al. (2014) exponen evidencia fenológica y morfológica suficiente para proponer que las poblaciones del oeste de Jalisco registradas antiguamente como $A$. guatemalensis var. jaliscana deben de ser elevadas al nivel de especie como A. jaliscana (Martínez) Mantilla, Shalisko \& A.Vázquez, siendo diferentes del resto de las poblaciones asignadas a A. flinckii (Rushforth, 1989). Ante esta evidencia se decidió modelar las poblaciones consideradas por Rushforth (1989) como A. flinckii (de aquí en adelante, A. flinckii sensu lato) y las poblaciones a A. jaliscana y A. flinckii (sensu Vázquez-García) por separado. Finalmente, se determinaron por separado los nichos de las dos variedades de A. duranguensis Martínez (A. durangensis var. coahuilensis y A. durangensis var. durangensis), que aunque a nivel genético son muy similares (Jaramillo-Correa et al., 2008; Aguirre-Planter et al., 2012), su distribución disyunta en las Sierras Madre Oriental y Occidental, respectivamente, podría indicar nichos ecológicos muy distintos. Cabe resaltar que una de las especies consideradas válidas por Farjon (2013), Abies hidalgensis Debreczy, I.Rácz \& Guízar, no se incluyó en el presente estudio por contar sólo con dos localidades conocidas; esto se reflejaría en modelos con un soporte estadístico muy pobre.

Obtención de los datos de presencia. Los registros de presencia de cada una de las especies se obtuvieron de diversas fuentes. La primera de ellas fue la base de datos de las colectas de Eguiarte y Furnier (1997), depositados en la CONABIO bajo la clave B138. Posteriormente se consultó el portal Global Biodiversity Information Facility ("GBIF"<http://www.gbif.org> consultado entre enero y diciembre de 2011), que incluye datos de herbarios de todo el mundo, cuyos registros fueron complementados con los del Herbario Nacional (MEXU) del Instituto de Biología de la Universidad Nacional Autónoma de México (UNAM). Algunos de los datos antiguos de esta última base de datos tuvieron que ser geo-referenciados conforme a los estándares delineados por Wieczorek et al. (2004) y al manual de procedimientos de geo-referenciación de localidades de CONABIO (Nuñez-Merchand, 2008) antes de ser incluidos en el presente estudio. Los datos obtenidos fueron depurados en varias fases antes de iniciar las modelaciones del nicho ecológico de cada taxón. En la primera de ellas se eliminaron los datos repetidos y aquellos cuya identidad taxonómica estaba en duda. Posteriormente, se excluyeron los datos mal georeferenciados y/o que representaban valores atípicos geográficos; es decir aquellos puntos en donde se reportó una especie de abeto, pero que se encuentran fuera de su distribución natural, como en costas o desiertos. Luego se excluyeron los valores atípicos climáticos; es decir, las localidades de colecta que se encuentran en zonas en las que es difícil determinar si constituyen o no parte de la distribución natural de la especie. Para ubicar estos valores atípicos se utilizaron dos métodos implementados en DIVA-GIS ver. 7.5 (Hijmans et al., 2012): (1) el método de Reverse jackknife (Chapman, 2005), que ha sido recomendado para conjuntos de datos con una distribución normal de valores (Scheldeman y van Zonneveld, 2011), como aquellos de muchas observaciones para cada taxón $(n>20)$ y (2) el método de $1.5 x$ Interquartile range (1.5 IQR; Scheldeman y van Zonneveld, 2011), que se recomienda para conjuntos de datos con poca cantidad de observaciones por taxón (por ejemplo, $\mathrm{n}<20$ ). Para todos los análisis se consideró que la cantidad mínima de variables en las que un punto de presencia debía tener valores extremos para ser considerado un punto atípico climático debía ser de tres (Scheldeman y van Zonneveld, 2011). Por último, para evitar problemas de sobre-modelación, los puntos de presencia se depuraron para dejar un sólo registro por celda (cada celda tenía 30 segundos de arco), lo cual se realizó utilizando la biblioteca Raster (Hijmans y van Etten, 2012) para el leguaje de programación R (R Core Team, 2014).

Obtención de datos ambientales y determinación del área accesible $(M)$. Se obtuvieron las 19 variables bioclimáticas de Worldclim (Hijmans et al., 2005), así como las capas de orientación, pendiente e índice topográfico de humedad (Wetness Index) de la base de datos geográfica Hydrolk (HYDRO1k Elevation Derivative Database) en formato Esri grid a una resolución espacial de 30 segundos de arco para el área correspondiente a México, Estados Unidos y Centroamérica. Estas capas fueron recortadas en áreas más pequeñas para ser utilizadas en los análisis de cada especie. El criterio para el recorte fue reducirlas al área accesible para cada especie o área $M$; es decir, aquellas áreas en donde cada especie está o se supone que podría estar dado el conocimiento biológico que se tiene de ellas, de sus capacidades de dispersión, y por no existir grandes barreras ni discontinuidades ambientales que pudieran limitar su establecimiento. El concepto de área accesible para una especie hace referencia al diagrama BAM propuesto por Soberón y Peterson (2005), este esquematiza que el área de distribución de esta especie en determinado tiempo es el resultado de una combinación de variables ambientales o scenopoeticas $(A)$, de variables bióticas $(B)$ y del área que la especie puede explorar $(M)$.

Para ayudar a determinar el área $M$ de cada especie se utilizaron las ecorregiones terrestres del mundo reportadas en Olson et al. (2004). Se escogieron aquellas que coincidían con la ubicación de los sitios de colecta para cada especie, evitando ecorregiones que aunque similares, no tuvieran registros. Esto se hizo siguiendo la lógica de que aunque la dispersión del polen y las semillas de Abies es por viento, la germinación de las semillas y el establecimiento de las plántulas se favorecen en condiciones más o menos sombreadas y al resguardo de otros miembros de la misma especie, sugiriendo que la posibilidad de colonizar zonas abiertas o exentas de bosque es baja (Williams, 2009). Las ecorregiones escogidas coinciden en su mayoría con zonas monta- 
ñosas y/o templadas donde prosperan bosques de coníferas apropiados para el establecimiento de los abetos (Enright y Hill, 1995). Solamente en dos casos (A. durangensis var. durangensis y $A$. vejarii) las poblaciones coincidieron con una sola ecorregión, para los otros, las fronteras entre ecorregiones contiguas se eliminó para tener una única área continua. Las áreas resultantes, que representan el área probablemente accesible $(M)$, se usaron como "clip" para recortar el conjunto de capas bioclimáticas a emplearse en el análisis de cada especie, luego de ser transformadas a formato ASCII. La selección y edición de las ecorregiones, así como el recorte y transformación de las capas bioclimáticas, se realizó utilizando el programa ArcMap10 (ESRI, 2011).

Reducción del conjunto de datos ambientales y extracción de información climática. Es sabido que utilizar una gran cantidad de capas bioclimáticas puede conducir a errores en las predicciones por sobreajuste de los modelos (Peterson y Nakazawa, 2008). Para evitar esto, se redujo el número de variables que mostraran multicolinealidad a partir de un análisis de correlación de Pearson realizado con los valores de cada pixel de cada una de las capas utilizadas, conservando sólo una de las variables altamente correlacionadas (correlación $\geq 0.8$ ). Este análisis se condujo por medio del programa ENMtools ver. 2.1 (Warren et al., 2010). En la medida de lo posible se escogieron aquellas variables que pudieran ser interpretadas más fácilmente, es decir aquellas como la "temperatura media anual" o la "precipitación anual", que conllevan un menor tratamiento matemático si se les compara con variables como la "oscilación anual de la temperatura", que es el resultado de la sustracción de las variables "máxima temperatura del mes más cálido" y "mínima temperatura del mes más frío". Cuando estos criterios no se cumplieron, las variables bioclimáticas a conservar se escogieron de manera aleatoria.

Modelado de nicho ecológico. Los análisis de distribución potencial se realizaron con el algoritmo de máxima entropía del programa Maxent ver. 3.3.3k. Este utiliza una técnica de aprendizaje de máquina para escoger los modelos más consistentes a partir la información disponible (Phillips et al., 2006). Este programa se escogió dado que se ha demostrado con distintas simulaciones que genera buenas predicciones, aun cuando se utilizan muestras pequeñas $(<10$; Phillips et al., 2006; Pearson et al., 2007). Los datos de presencia finales y las variables climáticas seleccionadas recortadas con el área $M$ correspondiente sirvieron de insumo para generar los modelos de nicho ecológico potencial para cada especie. Los parámetros de elaboración utilizados fueron aquellos que venían por omisión en el programa (Phillips y Dudik, 2008), a excepción de las opciones Extrapolate y Do clamping que se desactivaron para evitar extrapolaciones artificiales en los valores extremos de las variables ecológicas (Elith et al., 2011), dado que nuestras especies están restringidas a zonas montañosas.

Se obtuvo una salida de tipo logística usando un umbral de mínima presencia de entrenamiento (minimum training presence) que incluyó el $75 \%$ de los puntos para el entrenamiento del modelo y $25 \%$ para validar el mismo. Dichas validaciones se realizaron inicialmente con una prueba binomial, en la que se supone que los modelos son mejores que el azar cuando arrojan un valor de $P<0.01$. Posteriormente se utilizó el valor AUC (area under the curve) del análisis de ROC (receiver operating characteristic) para determinar la validez de los modelos. Los modelos con valores de AUC entre 0.7-0.9 para los puntos de entrenamiento y prueba se consideraron razonablemente buenos y los modelos con valores por arriba de 0.9 se catalogaron como muy buenos (Peterson et al., 2011). Sin embargo, es de notar que la utilidad de los análisis ROC ha sido cuestionada para los algoritmos que utilizan datos de solo presencia, ya que en teoría este análisis también requiere datos de ausencias verdaderas (y no solo de pseudoausencias), además de que pondera igual los errores de omisión que los de comisión (Lobo et al., 2007; Peterson et al., 2008). Por tales motivos, los modelos finales se validaron también por medio del análisis ROC parcial, que ha sido diseñado para tratar de subsanar estas deficiencias (Peterson et al., 2008). Este análisis se realizó con en el programa Tool for Partial-ROC (Narayani, 2008), utilizando un $50 \%$ de los puntos de evaluación independientes re-muestreados en 1,000 réplicas bootstrap y fijando un error de omisión no mayor al $5 \%$ (1-omission threshold $>0.95$ ), luego se realizó una prueba z para determinar si los valores de proporciones AUC parciales de los modelos de nicho fueron estadísticamente mejores que un modelo al azar (AUC $=1.0$ ). Los insumos para el análisis, o sea el número total de pixeles similares con sus valores de idoneidad y el valor de idoneidad para el pixel de cada sitio de presencia para cada análisis, así como los histogramas de frecuencias de las proporciones AUC para los valores de las distribuciones nulas arrojadas por Tool for Partial-ROC, fueron obtenidos con el programa $\mathrm{R}$ y la librería Raster. Los archivo tipo ASCII generados por el programa Maxent de los modelos de nicho ecológico para cada especie fueron proyectados posteriormente sobre un mapa del área de distribución correspondiente por medio del lenguaje de programación R (R Core Team 2014), utilizando las bibliotecas Raster (Hijmans y van Etten, 2012), Maptools (Bivand y Lewin-Koh, 2015)y Rworldmap (South, 2013).

Caracterización ambiental y diferencias entre nichos. Para determinar si existen diferencias entre los nichos de las especies estudiadas, particularmente aquellas que aún son fuente de controversias taxonómicas, se realizó un análisis de componentes principales (ACP) con los datos bioclimáticos y topográficos de todas las localidades para cada una de las especies incluidas en este estudio. Posteriormente los valores (scores) de los tres primeros ejes del ACP fueron utilizados para probar si existían diferencias significativas entre los nichos de las especies por medio de un análisis no paramétrico de varianza multivariado (MANOVA), ya 
que no cumplían con los requisitos de normalidad y homoscedasticidad. Los valores significativos de este MANOVA indican una separación de las especies en el espacio ambiental, por lo que posteriormente se realizó un análisis de Kruskal-Wallis para determinar en cuales de los tres ejes existía diferenciación entre las especies. Posteriormente se realizó una prueba post hoc de Gao, que es una prueba de comparaciones pareadas no paramétrica (Gao et al., 2008), para identificar los pares de especies estadísticamente diferentes en cada uno de los ejes. La extracción de los valores bioclimáticos y topográficos, así como los análisis estadísticos, se realizaron con la ayuda del lenguaje de programación $\mathrm{R}$ (R Core Team, 2014) utilizando las bibliotecas Raster (Hijmans y van Etten, 2012), Vegan (Oksanen et al., 2013) y Nparcomp (Konietschke, 2015).

Nichos ecológicos y Áreas Naturales Protegidas. Con el ánimo de contribuir a la conservación de los Abies a partir de nuestros resultados y tener datos para su posible manejo, se identificó el porcentaje de registros para cada especie y el área de los nichos ecológicos construidos con estos que coinciden con un área natural protegida en México. Para hacerlo primero se calculó el porcentaje de puntos de colecta para cada especie que están dentro de las poligonales de las áreas naturales protegidas federales y estatales (BezauryCreel et al., 2009). Después se calcularon para cada especie el número de pixeles por nivel de idoneidad ambiental (bajo, medio y alto) considerando un umbral de mínima presencia de entrenamiento de los modelos de nicho obtenidos, y se sobrepusieron a las poligonales de las áreas naturales protegidas federales y estatales, posteriormente se calculó el porcentaje de pixeles que quedaron dentro de estas áreas con respecto al total de pixeles por nivel de idoneidad ambiental.

La proyección de los nichos ecológicos en México, la sobreposición de las poligonales de las áreas naturales protegidas y el cálculo de los porcentajes de áreas de distribución potencial que están protegidos, totales y por nivel de idoneidad del hábitat, se realizó con ayuda del lenguaje de programación R (R Core Team, 2014) y las bibliotecas Raster (Hijmans y van Etten, 2012), Maptools (Bivand y Lewin-Koh, 2015).

\section{Resultados}

El número final de registros conservados para cada especie después del proceso de depuración de las bases de datos, y que fueron utilizados para modelar sus nichos ecológicos, se presentan en la Tabla 1. La mayor cantidad de datos eliminados fueron de sitios repetidos (incluyendo las localidades en un mismo pixel) o mal georreferenciados, siendo las especies con más datos suprimidos Abies concolor (con 406 de 536 registros iniciales), A. religiosa (284 de 398) y A. guatemalensis (200 de 259). De las otras especies se obtuvieron registros más depurados, dada su distribución restringida, por lo que prácticamente no se eliminaron datos. Los análisis de Reverse jackknife y de 1.5x Interquartile range (1.5 IQR) permitieron detectar sólo 29 localidades con valores climáticos atípicos para todas las especies (Tabla 2), siendo A. hickelii Flous \& Gaussen la especie que perdió más registros con este criterio (7) y A. durangensis var. coahuilensis ningún registro; las gráficas resultantes de dichos análisis se pueden solicitar a los autores. En promedio, se conservaron 52.25 registros por especie, siendo A. concolor el taxón con más localidades (127) y A. vejarii y A. durangensis var. coahuilensis las de menor representación (con 25 y 14, respectivamente). Estos últimos valores no son sorprendentes dada la rareza de estos taxa (Farjon, 1990; Farjon y Filer, 2013). La base depurada con la relación de coordenadas y nombres de las localidades, colectores y fechas de colecta empleadas para este estudio puede ser solicitada a los autores o consultada en formato DarwinCore en el portal de CONABIO ligada al proyecto JM015 (<http://www.conabio.gob.mx/institucion/cgi-bin/datos.cgi?Letras=JM\&Num ero=15>).

Tabla 1. Procedencia de los registros de Abies Mesoamericanos usados en la modelación de su nicho ecológico potencial.

\begin{tabular}{|c|c|c|c|c|}
\hline Especie & CONABIO & IBUNAM-MEXU & GBIF & Total \\
\hline Abies concolor (Gordon \& Glend.) Hildebr. & 0 & 0 & 127 & 127 \\
\hline Abies religiosa (Kunth) Schltdl. \& Cham. & 0 & 108 & 0 & 108 \\
\hline Abies guatemalensis Rehd. & 19 & 13 & 22 & 54 \\
\hline Abies flinckii Rushforth & 6 & 13 & $4(* 3)$ & 23 \\
\hline Abies flinckii s. I. & 6 & 29 & $6(* 5)$ & 41 \\
\hline Abies jaliscana (Martínez) Mantilla, Shalisko \& A. Vázquez & 0 & 16 & $2^{*}$ & 18 \\
\hline Abies hickelii Flous \& Gaussen & 9 & 14 & 15 & 38 \\
\hline Abies durangensis var. durangensis & 10 & 3 & 21 & 34 \\
\hline Abies durangensis var. coahuilensis (I. M. Johnst.) Martínez & 7 & 2 & 5 & 14 \\
\hline Abies vejarii Martínez & 5 & 7 & 13 & 25 \\
\hline
\end{tabular}

CONABIO: Comisión Nacional para el Conocimiento y Uso de la Biodiversidad.

IBUNAM-MEXU: Herbario Nacional de México bajo custodia del Instituto de Biología de la UNAM.

GBIF: Global Biodiversity Information Facility.

*En GBIF y en la base del INECOL. 
NoRberto MartíneZ-MÉNDEZ ET AL.

Tabla 2. Relación de puntos climáticos extremos excluidos de los análisis finales. Todos los puntos fueron determinados por el método de Reverse jackknife exceptuando el punto correspondiente a A. vejarii localizado por el método 1.5 IQR.

\begin{tabular}{|c|c|c|c|c|c|}
\hline Especie & $\begin{array}{l}\text { Código de la } \\
\text { institución o } \\
\text { base de datos }\end{array}$ & $\begin{array}{l}\text { Número de } \\
\text { catálogo }\end{array}$ & Colector & Longitud & Latitud \\
\hline \multirow[t]{3}{*}{ Abies concolor } & USDA NRCS & ABCO-06057 & $N D$ & -120.766 & 39.304 \\
\hline & USDA NRCS & ABCO-06023 & $N D$ & -123.912 & 40.7004 \\
\hline & USDA NRCS & ABCO-06015 & $N D$ & -123.957 & 41.7373 \\
\hline \multirow[t]{6}{*}{ Abies religiosa } & IBUNAM & 551509 & Rosa María Fonseca Juárez & 100.15225 & 17.47135 \\
\hline & IBUNAM & 649446 & J. Rzedowski & -100.2 & 17.48333 \\
\hline & IBUNAM & 1208924 & Jorge Calónico Soto & -99.83806 & 17.65111 \\
\hline & IBUNAM & 878768 & J. I. Calzada & -97.26667 & 19.05 \\
\hline & IBUNAM & 67666 & F. Miranda & -97.27878 & 18.98449 \\
\hline & IBUNAM & 259017 & J. Dorantes & -97.15653 & 19.50671 \\
\hline \multirow[t]{5}{*}{ Abies guatemalensis } & INECOL & K422946 & M. A. Carranza, P. Cruz A. \& D. Jonás & -100.833 & 27.017 \\
\hline & IBUNAM & 820456 & $N D$ & -98.94744 & 24.57709 \\
\hline & МOBOT & 3609257 & J. Hinton & -97.82 & 23.28 \\
\hline & FMNH & 1038435 & J. A. Steyermark & -90.1792 & 15.7422 \\
\hline & $\begin{array}{l}\text { CONABIO: Niveles y } \\
\text { patrones de variación } \\
\text { genética del género } \\
\text { Abies en México }\end{array}$ & ND & Glenn R. & -89.917 & 15.083 \\
\hline \multirow[t]{4}{*}{ Abies flinckii } & INECOL & 36791 & C. L. Díaz Luna & -103.39195 & 20.72028 \\
\hline & INECOL & 6400 & J. Rzedowski & -101.45667 & 19.235 \\
\hline & FITECMA-UMSNH & FITECMA 117 & M.S.X. \& G.G.L. & -101.5 & 19.85 \\
\hline & IBUNAM & 274506 & X. Madrigal Sánchez & -100.80127 & 19.67582 \\
\hline \multirow[t]{7}{*}{ Abies hickelii } & $\begin{array}{l}\text { CONABIO: Niveles } \\
\text { y patrones de } \\
\text { variación genética } \\
\text { del género Abies } \\
\text { en México }\end{array}$ & ND & Glenn R. & -97.45 & 19.533 \\
\hline & IBUNAM & 729332 & $N D$ & -97.15 & 19.5 \\
\hline & INECOL & V102299 & R. E. Arriaga C. & -97.125 & 19.46528 \\
\hline & IBUNAM & 945790 & $N D$ & -97.01127 & 19.41876 \\
\hline & IBUNAM & 773963 & $N D$ & -96.57877 & 17.07004 \\
\hline & IBUNAM & 1069871 & $N D$ & -96.56597 & 17.2521 \\
\hline & ND & 26213 & J. Hinton \& G. S. Hinton & -96.25889 & 16.18 \\
\hline \multirow[t]{3}{*}{$\begin{array}{l}\text { Abies durangensis } \\
\text { var. durangensis }\end{array}$} & CIIDIR-DURANGO & ND & $\begin{array}{l}\text { A. García Mendoza, B. T. Styles, } \\
\text { S. Favela, J. L. Campos \& M. González }\end{array}$ & -105.783 & 23.7 \\
\hline & UA & dbsn79669 & P.S. Martin & -108.55 & 27.79167 \\
\hline & $\begin{array}{l}\text { CONABIO: Niveles } \\
\text { y patrones de } \\
\text { variación genética } \\
\text { del género Abies } \\
\text { en México }\end{array}$ & ND & Glenn R. & -108.2 & 29.3 \\
\hline Abies vejarii & IBUNAM & 771820 & Robert T. Clausen & -99.47273 & 24.00015 \\
\hline
\end{tabular}

Los registros retenidos se distribuyeron en un total de 98 ecorregiones terrestres, siendo Abies concolor la especie que se distribuye en un mayor número de regiones (43), mientras que A. durangensis var. durangensis y A. vejarii únicamente estuvieron distribuidas en una ecorregión cada una. En el Apéndice 1 se puede consultar una relación de las ecorregiones terrestres del mundo utilizadas para obtener el área $M$ de cada especie.

En total se eligieron de cinco (Abies hickelii) a ocho capas (A. concolor y A. guatemalensis) de variables no-colineales para modelar el nicho de las diferentes especies de Abies mexicanos. La relación final de dichas capas se enlista 
en la Tabla 3. Las capas elegidas en más ocasiones fueron bio19 (precipitación del cuarto más frío), común para seis especies, seguida de elevación, bio2 (intervalo promedio de temperaturas diurnas), bio18 (precipitación del cuarto más cálido) y pendiente, que fueron compartidas por cinco especies. De este conjunto de variables, la altitud tuvo una alta correlación negativa $(<-0.9)$ con alguna o todas las capas relacionadas con temperatura (bio1 a bio11) en todas las especies, exceptuando a A. concolor para la cual las correlaciones negativas más altas fueron solo de -0.696 con bio11 (temperatura promedio del cuarto más frío) y de -0.692 con bio1 (temperatura media anual). Esto indica que la disminución de temperatura con el incremento de la altitud es más marcado en las zonas montañosas de áreas tropicales e in-

Tabla 3. Relación de las capas bioclimáticas y topográficas utilizadas para cada uno de los análisis finales de nicho ecológico.

\begin{tabular}{|c|c|}
\hline Especie & Capas utilizadas \\
\hline Abies concolor & $\begin{array}{l}\text { Altitud, } \mathrm{BIO} 2, \mathrm{BIO} 7, \mathrm{BIO} 15, \mathrm{BIO} 16, \mathrm{BIO} 17 \\
\mathrm{BlO} 18, \mathrm{BIO} 19\end{array}$ \\
\hline Abies religiosa & $\begin{array}{l}\text { Altitud, Pendiente, BIO2, BIO8, BIO11, } \\
\text { BIO15, BIO16 }\end{array}$ \\
\hline Abies guatemalensis & $\begin{array}{l}\text { Altitud, Orientación, } \mathrm{BIO} 2, \mathrm{BIO} 4, \mathrm{BIO} 5 \text {, } \\
\mathrm{BIO} \text {, BIO13, BIO18 }\end{array}$ \\
\hline Abies flinckii & $\begin{array}{l}\text { Orientación, BIO2, BlO6, BIO12, BlO14, } \\
\text { BlO15, BIO19 }\end{array}$ \\
\hline Abies flinckii s.l. & $\begin{array}{l}\text { Orientación, BIO2, BIO6, BIO12, BIO14, } \\
\text { BIO15, BIO19 }\end{array}$ \\
\hline Abies jaliscana & $\begin{array}{l}\text { Orientación, BIO1, BIO2, BlO4, BIO12, } \\
\text { BlO14, BlO15, }\end{array}$ \\
\hline Abies hickelii & Altitud, Orientación, BIO7, BIO17, BIO19 \\
\hline $\begin{array}{l}\text { Abies durangensis } \\
\text { var. durangensis }\end{array}$ & $\mathrm{BIO1}, \mathrm{BIO6}, \mathrm{BIO} 13, \mathrm{BIO} 15, \mathrm{BIO18,} \mathrm{BIO19}$ \\
\hline $\begin{array}{l}\text { Abies durangensis } \\
\text { var. coahuilensis }\end{array}$ & $\begin{array}{l}\text { Altitud, Orientación, Pendiente, BIO2, } \\
\text { BIO7, BIO18, BIO19 }\end{array}$ \\
\hline Abies vejarii & $\begin{array}{l}\text { Orientación, BIO6, BIO7, BIO17, BIO18, } \\
\text { BIO19 }\end{array}$ \\
\hline
\end{tabular}

Tabla 4. Valores de las proporciones AUC del análisis de ROC parcial.

\begin{tabular}{llll}
\hline Especie & $\begin{array}{l}\text { Proporciones AUC } \\
\text { del ROC parcial }\end{array}$ & DE* & $\begin{array}{c}\text { Prueba } \boldsymbol{Z} \\
\text { valor } \boldsymbol{P}\end{array}$ \\
\hline Abies concolor & 1.320 & \pm 0.051 & $<0.0001$ \\
Abies religiosa & 1.756 & \pm 0.063 & $<0.0001$ \\
Abies guatemalensis & 1.557 & \pm 0.125 & $<0.0001$ \\
Abies flinckii & 1.771 & \pm 0.118 & $<0.0001$ \\
Abies flinckii s.l. & 1.403 & \pm 0.172 & $<0.0001$ \\
Abies jaliscana & 1.769 & \pm 0.078 & $<0.0001$ \\
Abies hickelii & 1.640 & \pm 0.136 & $<0.0001$ \\
Abies durangensis & 1.456 & \pm 0.211 & $<0.0001$ \\
var. durangensis & & & \\
Abies durangensis & 1.724 & \pm 0.222 & $<0.0001$ \\
var. coahuilensis & & & \\
Abies vejarii & 1.522 & \pm 0.145 & $<0.0001$ \\
\hline
\end{tabular}

*Desviación estándar.

tertropicales, que en las zonas de latitudes extratropicales, como en el oeste de Estados Unidos, en donde se distribuye A. concolor. Las variables que se relacionaron con el aspecto del terreno (pendiente y orientación) no presentaron correlación alguna con ninguna de las demás variables y no parecieron ser tan importantes para caracterizar y marcar diferencias entre los nichos de las especies, con la excepción de A. durangensis var. coahuilensis (ver más adelante).

Los modelos de nicho ecológico para todas las especies analizadas (Figura 1) y para Abies finckii sensu lato (Figura 2) fueron mejores que el azar según la prueba binomial, que en todos los casos mostró valores significativos $(P<0.01)$. Estos modelos fueron igualmente catalogados como "muy buenos" según los valores de las pruebas ROC estándar, que fueron mayores a 0.900 para todas las especies. Finalmente, las pruebas de ROC parcial arrojaron razones de AUC con valores significativos $(P<0.0001)$ mayores a 1 (Tabla 4$)$, indicando nuevamente que los modelos obtenidos son estadísticamente mejores que el azar.

En general, las especies presentaron zonas de alta idoneidad $(>0.666)$ discontinuas y restringidas a ciertas zonas

Tabla 5. Sumario del análisis de multivariado de varianza (Manova) no paramétrico y del análisis de Kruskal-Wallis entre los tres primeros componentes del ACP.

\begin{tabular}{|c|c|c|c|c|c|c|}
\hline MANOVA r & g.l. & $\begin{array}{l}\text { Suma de } \\
\text { cuadrados }\end{array}$ & $\begin{array}{l}\text { Promedio de } \\
\text { cuadrados }\end{array}$ & Modelo $F$. & $R^{2}$ & $\operatorname{Pr}(>F)$ \\
\hline Especies & 9 & 0.72432 & 0.065847 & 56.431 & 0.52886 & 0.000999 \\
\hline Residuales & 553 & 0.64527 & 0.001167 & & 0.47114 & \\
\hline Total & 564 & 1.36958 & & & 1 & \\
\hline \multicolumn{2}{|c|}{ Prueba de suma de rangos de Kruskal-Wallis } & ji-cuadrada & valor $P$ & & & \\
\hline ACP1 & 10 & 270.1405 & $<2.2 \times 10^{-16}$ & & & \\
\hline ACP2 & 10 & 124.6733 & $<2.2 \times 10^{-16}$ & & & \\
\hline ACP3 & 10 & 162.5858 & $<2.2 \times 10^{-16}$ & & & \\
\hline
\end{tabular}



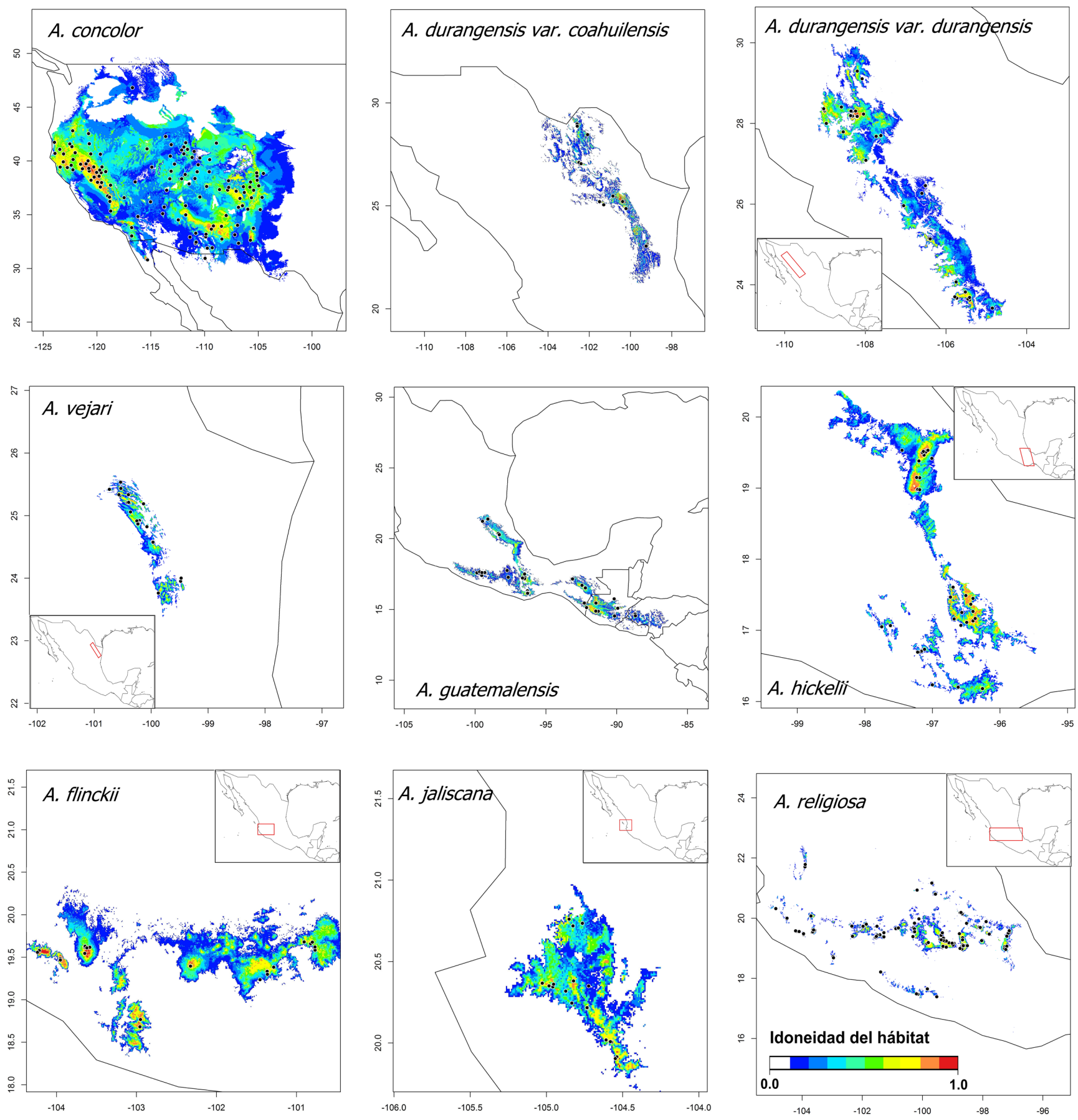

Figura 1. Proyección geográfica de los modelos de nicho ecológico para las especies de Abies de México y Centroamérica, incluyendo A. concolor. La escala de colores representa las áreas de idoneidad del hábitat, siendo mayor en los tonos más rojos.

montañosas (ver Figura 1). Sin embargo, hubo algunas diferencias entre taxa, pues para Abies concolor las poblaciones están en alturas que promedian los 1,670 m s.n.m., mientras que en las especies mesoamericanas las condiciones templadas adecuadas para los abetos (ver más adelante) sólo se alcanzan en alturas que promedian los 2,480 m s.n.m. No obstante, existen algunas poblaciones por debajo de los
1,000 m s.n.m., incluyendo 23 localidades de A. concolor, nueve de A. guatemalensis y dos de A. duranguensis var. coahuilensis, indicando que algunos sitios relativamente bajos también pueden tener condiciones climáticas adecuadas para los abetos.

El análisis de componentes principales (ACP) para todas las especies mostró que los tres primeros componentes 


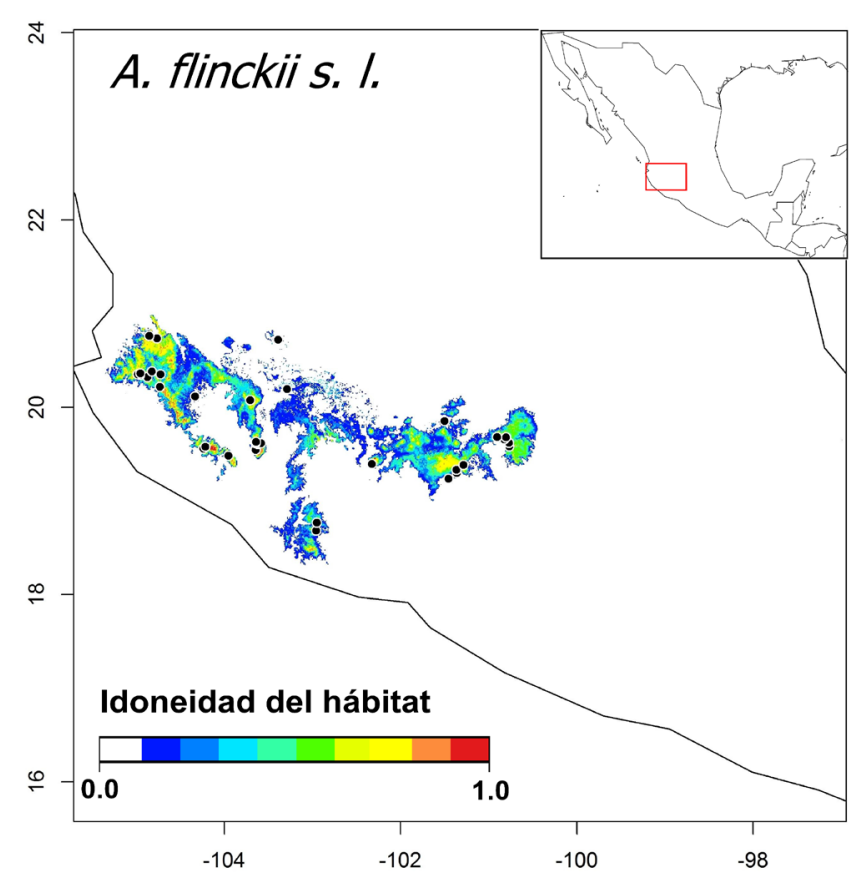

Figura 2. Proyección geográfica del modelo de nicho ecológico para Abies flinckii s. $l$. La escala de colores representa las áreas de idoneidad del hábitat, siendo mayor en los tonos más rojos. principales explican el $70 \%$ de la varianza (el $90 \%$ solo se alcanza hasta el séptimo componente) siendo cada uno de estos tres componente responsable del 40, 21.5 y $9 \%$ de la varianza, respectivamente. La gráfica de los tres primeros componentes (Figura 3 ) muestra que todas las especies están más o menos solapadas ambientalmente con excepción de Abies concolor, que es la especie que más se separa de las demás. Los resultados del MANOVA no paramétrico mostraron que al menos una especie es estadísticamente distinta $(F=56.431$, g.l. $=9, P=0.0009)$ en su espacio ambiental. Las pruebas de Kruskal-Wallis muestran que hay diferencias estadísticas entre las especies para cada uno de los tres primeros componentes principales (Tabla 5). Asimismo, la prueba de Gao (Apéndice 2) revela que en general la mayoría de las especies analizadas son similares entre sí con excepción de A. concolor, que para el primer componente (CP1) es la única especie que es estadísticamente distinta en sus atributos ambientales con respecto a todas las demás, aunque con los otros componentes muestra similitudes con A. durangensis var. coahulensis (CP2 y CP3) y con A. finckii (CP2).

Los datos crudos para todas las especies (datos no mostrados) indican que Abies concolor ocurre en las zonas con mayor estacionalidad de temperaturas (bio4) con un prome-

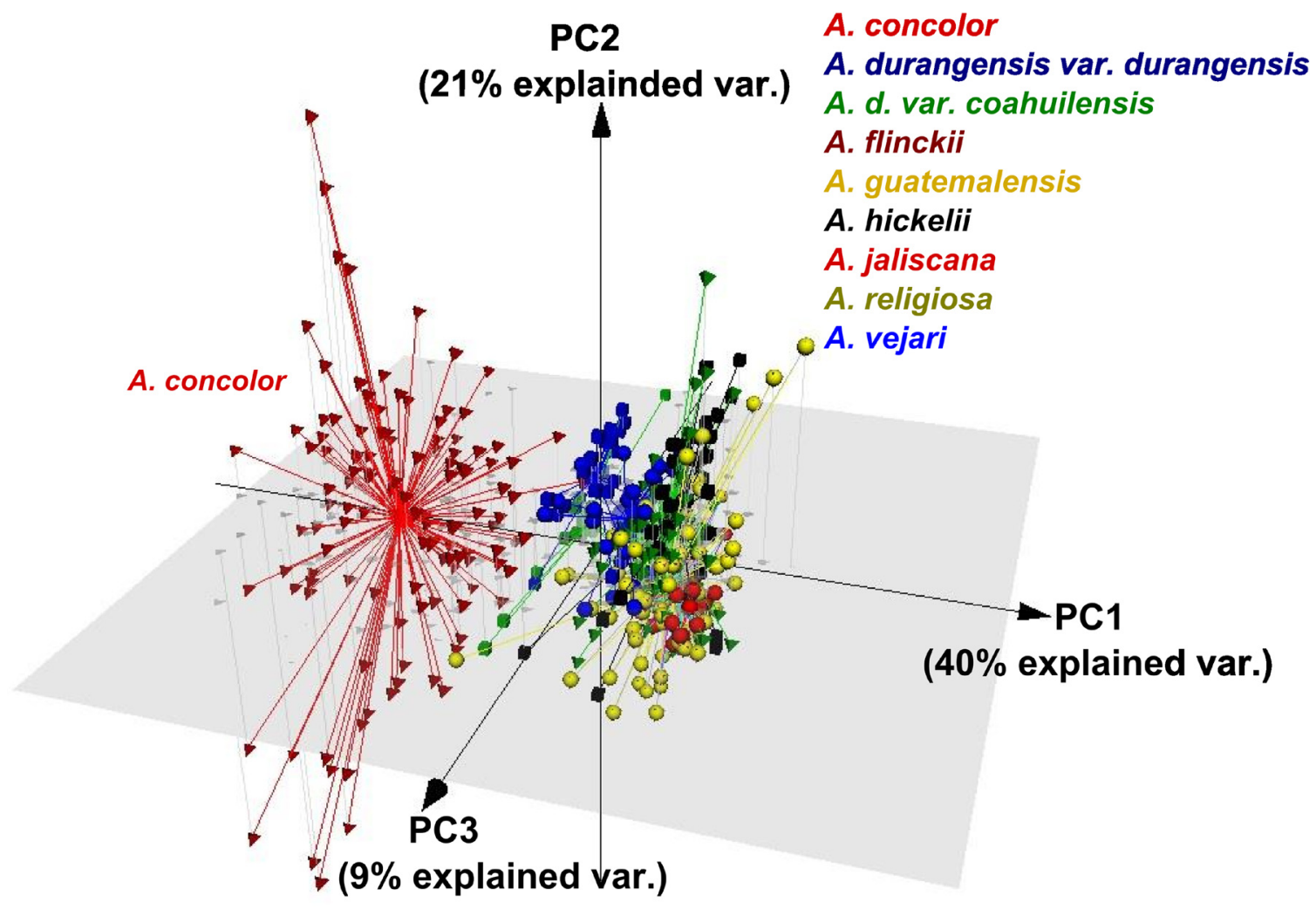

Figura 3. Análisis de componentes principales (ACP) de las variables bioclimáticas y topográficas que muestran las diferencias ecológicas entre las especies de abetos Mesoamericanos. Los puntos correspondientes de Abies flinckii s.l. no se marcaron de color ya que se superponen con los de A. flinckii y con los de A. jaliscana. 
dio para todos los sitos de $9.5^{\circ} \mathrm{C}$. Por otra parte, los demás abetos se distribuyen en zonas con una estacionalidad de temperatura menos marcada y un poco más cálidas, con temperaturas promedio anuales que van de $\operatorname{los} 12^{\circ} \mathrm{C}$ en las localidades de A. religiosa a los $16.4{ }^{\circ} \mathrm{C}$ en las poblaciones de A. guatemalensis. La precipitación a lo largo del área de distribución de A. concolor también es muy distinta a la de los demás taxa mexicanos, con la mayor cantidad de humedad concentrada alrededor de los meses más fríos (bio16 y bio19) y con un promedio de $554 \mathrm{~mm}$ por año, mientras que para los otras especies la precipitación está concentrada en los meses de verano o repartida a lo largo del año y con promedios anuales que van de $598 \mathrm{~mm}$ para A. durangensis var. coahuilensis a $1,322 \mathrm{~mm}$ para A. hickelii.

Cuando se sobrepusieron las poligonales de las zonas naturales protegidas estatales y federales a los modelos de nicho ecológico de las especies de abeto presentes en México, se observó que el porcentaje de colectas y de las zonas de mayor idoneidad ambiental (>0.6) que está dentro de un área natural protegida (ANP) federal o estatal varía mucho con cada especie, pero en ningún caso es del $100 \%$ (Apéndice 3). En general, las especies con una distribución geográfica menor fueron las que mostraron mayor porcentaje de registros y de áreas de alta idoneidad protegidas, como Abies vejarii con un 56 y $76.16 \%$ o Abies durangensis var. coahuilensis con un 78.57 y $63.65 \%$ respectivamente. En el caso de A. concolor, ningún punto de colecta en México cae dentro de una ANP, aunque de la fracción de nicho ecológico potencial de esta especie presente en México (3.1\%) el porcentaje de áreas con alta idoneidad que pudieran estar protegidas dentro de una ANP es de un $63.87 \%$. En el caso de A. religiosa, una de las especies con mayor distribución en el Faja Volcánica Transmexicana, el porcentaje de puntos de colecta y de áreas con alta idoneidad protegidas es de un $41.67 \%$ y un $60.9 \%$ respectivamente, mientras que para otras especies en la Faja Volcánica Transmexicana como A. flinckii, A. flinckii s. l. y A. hickelii este porcentaje es más bajo y corresponde al $26.09 \%$ y $33.2,19.51 \%$ y 31.94 y al $7.89 \%$ y $24.3 \%$, respectivamente; para A. jaliscana se registran $11.11 \%$ de colectas dentro de una ANP, pero sólo el $1.96 \%$ de su distribución potencial coincidió con una, constituyéndose en la especie menos protegida del país. La siguiente especie menos protegida es A. guatemalensis, con sólo $8.16 \%$ de registros y un $8.9 \%$ de áreas de alta idoneidad ambiental dentro de una ANP. Cabe anotar que paradójicamente, esta especie es la más abundante de Mesoamérica con un $83.7 \%$ de registros y un $78.6 \%$ de su distribución potencial ubicada en México. Otra especie que también tiene una baja protección es A. durangensis var. durangensis, que presenta solo el $20.59 \%$ de los registros dentro de una ANP con áreas de alta idoneidad muy fragmentadas y alejadas sobre la Sierra Madre Oriental y de las cuales solo el $17.45 \%$ estaría protegida.

\section{Discusión}

Este estudio es el primero en determinar y comparar el nicho ecológico potencial de la mayoría de las especies del género Abies en México. Los modelos de nicho ecológico obtenidos presentaron un desempeño alto de acuerdo a los resultados de las pruebas binomial, de ROC y ROC parcial (Peterson et al., 2008). Esto se vio corroborado en el hecho de que para todas las especies las zonas con mayor idoneidad ambiental fueron disyuntas y estuvieron limitadas a las partes altas de las sierras coincidiendo con los conocimientos básicos de la biología de las mismas (p.ej. Farjon, 2013). Los análisis multivariados derivados de los resultados de estos modelos mostraron que la especie ecológicamente más divergente fue $A$. concolor mientras que los taxa restantes comparten características ecológicas similares. Es interesante anotar que A. concolor ya había mostrado una divergencia significativa a nivel genético (Aguirre-Planter et al., 2012), lo que sugiere que para esta especie, la diferenciación evolutiva y ecológica parecen estar correlacionadas (Van Valen, 1976; ver también Aguirre-Planter et al., 2012 para una discusión al respecto). Estudios similares con marcadores nucleares (Aguirre-Planter et al., 2000; Múgica-Gallart, 2013) y de cloroplasto (Jaramillo-Correa et al., 2008) también habían indicado que A. flinckii s.l. también es una entidad genética independiente de las demás especies de Abies en México, pero los resultados indican que esta especie es poco distinguible ecológicamente del resto de sus congéneres. Esta misma conclusión se puede ampliar a A. jaliscana y A. flinckii s.s., las dos especies que Vázquez-García y colaboradores reconocen como diferentes a partir de datos morfológicos. Estas similitudes podrían sugerir una evolución por conservadurismo de nicho para las especies de este gran clado, aunque esta hipótesis aún deberá ser probada en estudios futuros y tal vez integrada a análisis filogenéticos con un mayor número de marcadores nucleares.

Volviendo a Abies concolor, esta especie se encuentra actualmente en pocas poblaciones en el norte de México, con la mayor parte de su distribución en grandes áreas del oeste y sudoeste de los Estados Unidos, en regiones que se caracterizan por tener inviernos fríos y precipitaciones concentradas durante el trimestre más frío del año. Las demás especies de abetos mexicanos están distribuidas en zonas con inviernos más templados y con las precipitaciones más altas concentradas en el trimestre más cálido del año. La divergencia genética que se ha observado entre $A$. concolor y los demás Abies mesoamericanos podría entonces estar relacionada con adaptaciones a estos factores ambientales, que ya han sido señalados como motores importantes en la evolución de otras coníferas (p. ej. Eckert et al., 2010; Mosca et al., 2012; Prunier et al., 2012). Sin embargo, esto también deberá ser probado con estudios genómicos de asociación similares a los efectuados en coníferas modelo 
como Pinus taeda o Picea glauca (Eckert et al., 2010; Prunier et al., 2012).

Las similitudes entre los nichos ecológicos de las demás especies de abeto de México son probablemente un reflejo de su origen relativamente reciente y la falta de monofilia recíproca (ver Aguirre-Planter et al., 2012; Xiang et al., 2015). Ésta se explica por probables ciclos de aislamiento y contacto secundario entre poblaciones y especies después de la migración del género hacia Mesoamérica entre el Mioceno y Plioceno (Eguiarte y Furnier, 1997; Graham, 1999; Aguirre-Planter et al., 2000, 2012). De esta manera, los abetos mesoamericanos parecen encontrarse en una "zona gris de especiación" (de Queiroz, 1998, 2005), en donde, debido al reciente tiempo de separación de las poblaciones, la monofilia recíproca es incompleta y las poblaciones pueden no formar grupos fenotípicamente y ecológicamente distinguibles o diagnosticables, por lo que varios conceptos de especie entran en conflicto y no hay un consenso sobre cuantas especies existen realmente. Los ciclos de aislamiento y contacto arriba mencionados probablemente estuvieron mediados por el "seguimiento ecológico" que las poblaciones de abetos hicieron de las condiciones templadas características de sus nichos. Este seguimiento ecológico parece haber influenciado los patrones genéticos y de distribución de poblaciones de muchas otras coníferas (Gugger et al., 2011; Moreno-Letelier et al., 2013) y ha sido sustentado con datos palinológicos que indican que los bosques templados de Mesoamérica se extendieron por debajo de los 1,000 m s.n.m. durante el último máximo glacial hace unos $21 \pm 2$ ka (Bush et al., 2009). De esta forma, durante las fases de expansión en el pasado, los bosques de coníferas aumentaron sus áreas, facilitando el intercambio de genes entre poblaciones y especies, mientras que en las fases más cálidas (como el presente interglacial), las poblaciones se fragmentaron y se desplazaron hacia las zonas más altas y templadas (Ramírez-Barahona y Eguiarte-Fruns, 2013).

El empleo de nuevos algoritmos y aproximaciones estadísticas, como la utilización de kernels de suavización acoplados a análisis de solapamiento de nicho ecológico para evitar errores por sesgos en los muestreos (Broennimann et al., 2012), además de la utilización de capas a mayor resolución y otras generadas a partir de trabajos de percepción remota, podrían mejorar la resolución de las diferencias ecológicas y climáticas en las especies estudiadas. Por lo que se espera que los resultados de dichos análisis puedan ser integrados en futuros estudios comparativos a nivel filogenético-ecológico para los Abies mexicanos. Por el momento, los resultados ecológicos, genéticos y morfométricos disponibles para este género en el país parecen indicar que la taxonomía tradicional basada en caracteres morfológicos ha conducido a confusiones debido a una elevada plasticidad fenotípica en muchos taxa (De Kroon et al., 2005). Por ejemplo, en un estudio previo ya se había mostrado que existe una gran similitud morfométrica entre las especies de
Abies aquí estudiadas, hasta el punto de ser virtualmente indistinguibles (Strandby et al., 2009). Desde este punto de vista, parece indispensable incorporar nuevos métodos para afinar las descripciones taxonómicas disponibles, como las simulaciones de nicho ecológico, el uso de marcadores moleculares y el establecimiento de ensayos de procedencias en campo, en donde se pueda estimar plasticidad fenotípica de las especies de forma adecuada.

Los estudios sobre modelado de nicho ecológico de coníferas, y en especial de abetos en México, son pocos. Uno de los primeros estudios sobre este tema fue el de Téllez-Valdés et al. (2004) en el cual se exploraba el modelado bioclimático como una herramienta de manejo forestal en cuatro especies de pino de importancia comercial. Posteriormente, Sáenz-Romero et al. (2012) modelaron el nicho ecológico de Abies religiosa en relación con su importancia como refugio de la mariposa monarca y las afectaciones ante distintos escenarios de cambio climático. Los otros estudios existentes se enfocan en utilizar el modelado de nicho ecológico como herramienta auxiliar para explicar algunos patrones filogeográficos (Gugger et al., 2011; Moreno-Letelier et al., 2013). Es así que la importancia de este trabajo radica en que los datos aquí generados son los primeros en comparar ecológicamente a casi todas las especies de abetos en México y nos ayudarán a poner a prueba distintas hipótesis filogeográficas y al mismo tiempo obtener datos de partida para estudios de conservación y cambio climático (ver más adelante).

Áreas Naturales Protegidas y conservación de abetos en México. Actualmente, la mayoría de las zonas de mayor idoneidad ambiental para los abetos mexicanos están fuera de las áreas naturales protegidas (ANP), con excepción de Abies religiosa y de especies con distribuciones geográficas restringidas, como A. vejarii o A. duranguensis var. coahuilensis. Lo anterior coincide con otros estudios que muestran que en México las ANP resguardan una proporción muy baja de especies $(<20 \%)$, sobre todo las de aquellas en alguna categoría de riesgo (Londoño-Murcia y SánchezCordero, 2011), que sólo tienen un pequeño porcentaje de su distribución potencial dentro de un ANP (Contreras-Medina et al., 2010). Es de resaltar que, dado que los nichos de los Abies mexicanos son muy estrechos y que los niveles de diferenciación genética nuclear entre poblaciones son muy altos (Eguiarte y Furnier, 1997; Aguirre-Planter et al., 2000), se debe tratar de conservar la mayor parte de éstas como se propone para el género Agave en México (Eguiarte et al., 2013). En este sentido, sería recomendable generar nuevas áreas naturales protegidas que incluyan las zonas de mayor idoneidad ambiental, especialmente para los taxa poco protegidos, como A. guatemalensis y A.durangensis var. durangensis.

Un caso especial es el de Abies finckii y A. jaliscana, ya que según la NOM059-SEMARNAT-2010 (SEMARNAT, 2010) A. flinckii es una especie en peligro. Sin embargo, la nueva propuesta de reconocer como una especie válida $(A$. 
jaliscana) a las poblaciones más occidentales de A. flinckii s.l. (Vázquez-García et al., 2014), deja a A. flinckii en un estado aun más vulnerable de lo que se había presupuestado y A. jaliscana casi sin protección (al menos en un sentido estricto). Este último punto se ve agravado por el hecho de que esta nueva especie es la que tiene una menor área potencial dentro de una ANP, por lo que sería de especial interés ampliar la zonas de protección para esta especie y para A. flinckii (independientemente del estatus taxonómico de cada una). Esta sugerencia se ve reforzada por la clara diferenciación genética que estos dos taxa en su conjunto (A. flinckii s.l.) muestran con el resto de abetos de Mesoamérica. Por otro lado, sería urgente realizar estudios genéticos y ecológicos sobre A. hidalgensis para verificar su estatus taxonómico y, si es el caso, resguardar sus poblaciones. En este sentido serían necesarias nuevas exploraciones en la zona donde se distribuye (Hidalgo, cerca de Metepec) para aumentar el número de localidades disponibles en bases de datos públicas. Debe recordarse que este taxón fue excluido del presente estudio, pues apenas se contó con dos localidades (ver Debreczy y Rácz, 1995) en dichas bases, lo que es insuficiente para desarrollar modelos de nicho adecuados.

Debido al acelerado calentamiento global (Zeng et al., 2004; Peng et al., 2014), es muy probable que las zonas de mayor idoneidad ambiental para los Abies comiencen a diferir de las encontradas en este estudio (ver Sáenz-Romero et al., 2012). Actualmente, ya se han registrado cambios en el tamaño y localización de las zonas de idoneidad ambiental para muchas plantas en todo el mundo, registrándose cambios más severos en zonas montañosas, donde muchas especies tienden a elevar su límite altitudinal dependiendo la especie y la zona geográfica (Wilson et al., 2005; Lenoir et al., 2008; Parolo y Rossi, 2008; Telwala et al., 2013). De esta forma, es urgente realizar análisis con diferentes escenarios de cambio climático para determinar cuáles serán estás nuevas zonas de idoneidad ambiental y determinar cuáles de los poblaciones actuales están en riesgo de desaparecer. Con estos datos se podrían proponer nuevos límites para las áreas naturales protegidas y tratar de implementar programas de rescate para estas especies. A este respecto, Sáenz-Romero et al. (2012) propusieron para A. religiosa que la migración asistida a zonas de mayor altitud e idoneidad ambiental debe ser una estrategia a considerarse. Para las demás especies de abeto se podrían sugerir estrategias similares; sin embargo, estudios más detallados sobre la diversidad genética, la plasticidad fenotípica y el potencial evolutivo de cada población y especie son necesarios para poder mejorar los programas de conservación existentes (Benito-Garzón et al., 2011).

\section{Agradecimientos}

Los autores agradecen el financiamiento de la Comisión Nacional para el conocimiento de la biodiversidad (CO-
NABIO) de México a través del proyecto JM015 "Modelación de la distribución geográfica de las especies del género Abies presentes en México" y a la beca posdoctoral de la Coordinación de la Investigación Científica de la UNAM otorgada al Dr. Norberto Martínez-Méndez que permitió la realización de este trabajo. Asimismo, agradecemos a los revisores por los valiosos comentarios y observaciones que mejoraron y enriquecieron de manera sustancial el trabajo.

\section{Literatura citada}

Aguirre-Planter E., Furnier G.R. y Eguiarte L.E. 2000. Low levels of genetic differentiation among populations of species of Abies from southern Mexico and Guatemala. American Journal of Botany 87:362-371.

Aguirre-Planter E., Jaramillo-Correa J.P., Gómez-Acevedo S., Khasa D.P., Bousquet J. y Eguiarte L.E. 2012. Phylogeny, diversification rates and species boundaries of Mesoamerican firs (Abies, Pinaceae) in a genus-wide context. Molecular Phylogenetics and Evolution 62:263-274.

Alvarado D., de Bauer L. y Galindo J.A. 1991. Declinación y muerte de bosque de oyamel (Abies religiosa) en el sur del Valle de México. Agrociencia, serie Recursos Naturales Renovables 3:123-143.

Benito-Garzón M., Alía R., Robson T.M. y Zavala M.A. 2011. Intra-specific variability and plasticity influence potential tree species distributions under climate change. Global Ecology and Biogeography 20:766-778.

Bezaury-Creel J.E., Torres J.F., Ochoa-Ochoa L.M., Castro-Campos M., Moreno N. 2009. Base de Datos Geográfica de Áreas Naturales Protegidas Estatales, del Distrito Federal y Municipales de México - Versión 2.0, Julio 31, 2009 < http://www.conabio.gob.mx/informacion/gis/> (consultado en mayo de 2014).

Bivand R. y Lewin-Koh N. 2015. maptools: Tools for Reading and Handling Spatial Objects. R package version 08.37.

Bush M.B., Correa-Metrio A.Y., Hodell D.A., Brenner M., Anselmetti F.S., Ariztegui D., Mueller A.D., Curtis J.H., Grzesik D.A., Burton C. y Gilli A. 2009. Re-evaluation of climate change in lowland Central America during the last glacial maximum using new sediment cores from lake Petén Itzá, Guatemala. En: Vimeaux F., Sylvestre F. y Khodri M. Eds. Past Climate Variability in South America and Surrounding Regions: From the Last Glacial Maximum to the Holocene, pp. 113-128, Developments in Paleoenvironmental Research Volume 14, Springer.

Broennimann O., Fitzpatrick M.C., Pearman P.B., Petitpierre B., Pellissier L., Yoccoz N.G., Thuiller W., Fortin M.-J., Randin C., Zimmermann N.E., Graham C.H. y Guisan A. 2012. Measuring ecological niche overlap from occurrence and spatial environmental data. Global Ecology and Biogeography 21:481-497.

Cayuela L. 2006. Deforestación y fragmentación de bosques tropicales montanos en los altos de Chiapas, México. Efectos sobre la diversidad de árboles. Ecosistemas 15:192-198.

Chapman A.D. 2005. Principles and Methods of Data Cleaning Primary Species and Species-Occurrence Data, version 1.0. Report for the Global Biodiversity Information Facility, Copenhagen. Disponible en: <http://www.gbif.org/orc/?doc_id=1262> (consultado en mayo de 2014).

CONAFOR [Comisión Nacional Forestal]. 2012. Inventario Na- 
cional Forestal y de Suelos. Informe 2004-2009. Coordinación General de Planeación e Información a través de la Gerencia de Inventario Forestal y Geomática. Comisión Nacional Forestal, Secretaria de Medio Ambiente y Recursos Naturales Zapopan,. Disponible en: <http://www.ccmss.org.mx/descargas/Inventario_nacional_forestal_y_de_suelos_informe_2004_-_2009_ .pdf $>$ (consultado en mayo de 2014).

CONAP [Comisión Natural de Áreas Naturales Protegidas]. Áreas Naturales Protegidas Federales de México. < http://sig. conanp.gob.mx/website/anpsig/viewer2.htm > (consultado en mayo de 2014).

Contreras-Medina R., Luna-Vega I. y Ríos-Muñoz C.A. 2010. Distribución de Taxus globosa (Taxaceae) en México: Modelos ecológicos de nicho, efectos del cambio del uso de suelo y conservación. Revista Chilena de Historia Natural 83:421-433.

Cuevas-Guzmán R., Cisneros-Lepe E.A., Jardel-Peláez E.J., Sánchez-Rodríguez E.V., Guzmán-Hernández L., Núñez-López N.M. y Rodríguez-Guerrero C. 2011. Análisis estructural y de diversidad en los bosques de Abies de Jalisco, México. Revista Mexicana de Biodiversidad 82: 1219-1233.

Debreczy Z. y Rácz I. 1995. New species and varieties of conifers from México. Phytologia 78: 217-243.

De Kroon H., Huber H., Stuefer J.F. y Van Groenendael J.M. 2005. A modular concept of phenotypic plasticity in plants. New Phytologist 166:73-82.

de Queiroz K. 1998. The general lineage concept of species, species criteria, and the process of speciation: A conceptual unification and terminological recommendations. En: Howard D.J, y Berlocher S.H. Eds. Endless forms: Species and Speciation, pp 57-75, Oxford University Press, Oxford.

de Queiroz K. 2005. A unified concept of species and its consequences for the future of taxonomy. Proceedings of the California Academy of Sciences 56:196-215.

Eguiarte L.E. y Furnier G.R. 1997. Niveles y patrones de variación genética del género Abies en México. Universidad Nacional Autónoma de México. Instituto de Ecología. Bases de datos SNIB2010-CONABIO proyecto No. B138. México, D.F.

Eguiarte L.E., Aguirre-Planter E., Aguirre X., Colín R., González A., Rocha M., Scheinvar E., Trejo L. y Souza V. 2013. From isozymes to genomics: Population genetics and conservation of Agave in Mexico. The Botanical Review 79:483-506.

Eckert A.J., van Heerwaarden J., Wegrzyn J.L., Nelson C.D., RossIbarra J., González-Martínez S.C. y Neale D.B. 2010. Patterns of population structure and environmental associations to aridity across the range of loblolly pine (Pinus taeda L., Pinaceae). Genetics 185:969-982.

Elith J., Phillips S.J, Hastie T., Dudík M., Chee Y.E. y Yates C.J. 2011. A statistical explanation of MaxEnt for ecologist. Diversity and Distributions 17:43-57.

Enright N.J. y Hill R.S. 1995. Ecology of the Southern Conifers. Smithsonian Institute, Washington D.C.

ESRI. 2011. ArcGIS Desktop: Release 10. Redlands, CA: Environmental Systems Research Institute.

Farjon A. 1990. Pinaceae: Drawings and Descriptions of the Genera Abies, Cedrus, Pseudolarix, Keteleeria, Nothotsuga, Tsuga, Cathaya, Pseudotsuga, Larix and Picea. Koeltz Scientific Books, Köenigstein.

Farjon A. 2010. A Handbook of the World's Conifers, vol.1. Brill Academic Publishers, Leiden.

Farjon A. 2013. Abies durangensis. The IUCN Red List of Threa- tened Species 2013: e.T42279A2969264. <http://dx.doi. org/10.2305/IUCN.UK.2013-1.RLTS.T42279A2969264.en.> (consultado en mayo de 2014).

Farjon A. 2013. Abies hidalgensis. The IUCN Red List of Threatened Species 2013: e.T42554A2987721. <http://dx.doi. org/10.2305/IUCN.UK.2013-1.RLTS.T42554A2987721.en.> (consultado en mayo de 2014).

Farjon A. 2013. Abies religiosa. The IUCN Red List of Threatened Species 2013: e.T39592A2929657. <http://dx.doi.org/10.2305/ IUCN.UK.2013-1.RLTS.T39592A2929657.en.> (consultado en mayo de 2014).

Farjon A. y Rushforth K.D. 1989. A classification of Abies Miller (Pinaceae). Notes from the Royal Botanical Garden Edinburgh 46: $59-79$.

Farjon A. y Filer D. 2013. An atlas of the world's conifers: An analysis of their distribution, biogeography, diversity, and conservation status. Brill Academic Publishers, Leiden.

Gao X., Alvo M., Chen J. y Li G. 2008. Nonparametric multiple comparison procedures for unbalanced one-way factorial designs. Journal of Statistical Planning 138: 2574-2591.

GBIF (Global Biodiversity Information Facility). 2011. Gobierno de Dinamarca. Disponible en: <http://www.gbif.org/ resources/2970> (consultado entre enero y diciembre de 2011).

Gómez-Gómez L.E. 2004. Valoración de bienes y servicios ambientales en los bosques naturales de pinabete (Abies guatemalensis Rehder), en tres regímenes de propiedad, de los municipios San José Ojetenam, San Cristóbal Ixchiguán y Concepción Tutuapa, San Marcos. Tesis Ingeniería Forestal, Centro Universitario del Nor Occidente, Universidad San Carlos, Guatemala, Guatemala. 110 pp.

Graham A. 1999. The tertiary history of the northern temperate element in the northern Latin American biota. American Journal of Botany 86: 32-38.

Gugger P.F., González-Rodríguez A., Rodríguez-Correa H., Shinya Sugita y Cavender-Bares J. 2011. Southward Pleistocene migration of Douglas-fir into Mexico: phylogeography, ecological niche modeling, and conservation of 'rear edge' populations. New Phytologist 189: 1185-1199.

Hijmans R.J., Cameron S.E., Parra J.L., Jones P.G. y Jarvis A. 2005. Very high resolution interpolated climate surfaces for global land areas. International Journal of Climatology 25:19651978.

Hijmans R.J., Guarino L. y Mathur P., 2012. DIVA-GIS. Ver. 7.5. A geographic information system for the analysis of species distribution data.

Hijmans R.J., Guarino L. y Mathur P. 2012. DIVA-GIS versión 7.5 User Manual. <http://www.diva-gis.org/docs/DIVA-GIS_ manu> (consultado en diciembre de 2012).

Hijmans R.J. y van Etten J. 2012. raster: Geographic analysis and modeling with raster data. $\mathrm{R}$ package version 2.0-12.

HYDRO1k Elevation Derivative Database. Earth Resources Observations and Science. <http://eros.usgs.gov/\#/Find_Data/Products_and_Data_Available/gtopo30/hydro/namerica> (consultado en diciembre de 2012)

IUCN [International Union for Conservation of Nature]. 2011. IUCN Red List of Threatened Species. Version 2011.1.<www. iucnredlist.org>. (Consultado el 19 de Agosto del 2011)

Jaramillo-Correa J.P., Aguirre-Planter E., Khasa D.M, Eguiarte L.E., Piñero D., Furnier G.R. y Bousquet J. 2008. Ancestry and divergence of subtropical montane forest isolates: molecular 
biogeography of the genus Abies (Pinaceae) in southern México and Guatemala. Molecular Ecology 17:2476-2490.

Konietschke F. 2015. nparcomp: Multiple Comparisons and Simultaneous Confidence Intervals. R package version 2.0-12.

Lenoir J., Gegout J.C, Marquet P.A, de Ruffray P. y Brisse H. 2008. A significant upward shift in plant species optimum elevation during the 20th century. Science 320:1768-1771.

Liu T.S. 1971. Department of Forestry, College of Agriculture, National Taiwan University, Taipei.

Lobo J.M., Jiménez-Valverde A. y Real R. 2007. AUC: a misleading measure of the performance of predictive distribution models. Global Ecology and Biogeography 17:145-151.

Londoño-Murcia M.C. y Sánchez-Cordero V. 2011. Distribución y conservación de especies amenazadas en Mesoamérica, Chocó y Andes tropicales. Revista Mexicana de Biodiversidad 82:926-950.

Narayani B. 2008. Tool for Partial-ROC (Biodiversity Institute, Lawrence, KS), ver 1.0.

Nuñez-Merchand A., Muñoz-López E., Hernández-Barrios J.C., Márquez-Mendoza J.D., Colín-López J.J., Jiménez-Cruz M., Moreno-Almeraya N., Herrera-López O. y López-Mendoza R. 2008. Georreferenciación de localidades de Colecciones Biológicas. Manual de Procedimientos. Comisión Nacional para el Conocimiento y Uso de la Biodiversidad, México D.F. Disponible en: <http:// www.biodiversidad.gob.mx/region/pdf/Manual\%20Georref_Vr1. pdf $>$ (consultado en diciembre de 2012).

Manzanilla H. 1974. Investigaciones Epidométricas y Silvícolas en Bosques Mexicanos de Abies religiosa. Dirección General de Información y Relaciones Públicas de la SAG. México, D.F.

Martínez-Arévalo J.V. 2013. Plantas asociadas a los bosques de Abies guatemalensis (Pinaceae) del occidente de Guatemala. Revista de Biología Tropical 61:321-333.

Martínez M. 1948. Los Abies mexicanos. Anales del Instituto de Biología de México 19:11-104.

Moreno-Letelier A., Ortíz-Medrano A. y Piñero D. 2013. Niche divergence versus neutral processes: Combined environmental and genetic analyses identify contrasting patterns of differentiation in recently diverged pine species. PLOS ONE 8:e78228.

Mosca E., Eckert A.J., Di Pierro E., Rocchini D., La Porta N., Belletti P. y Neale D.B. 2012. The geographical and environmental determinants of genetic diversity for four alpine conifers of the European Alps. Molecular Ecology 21:5530-5545.

Múgica-Gallart, J. 2013. Patrones de diversidad y detección de grupos genéticos en dos especies de Abies con distribuciones contrastantes en el Eje volcánico Transmexicano.Tesis de licenciatura, Facultad de Ciencias, Universidad Nacional Autónoma de México, México, D.F. 93 pp.

Oksanen J., Blanchet F.G. y Kindt R. 2013. vegan: Community Ecology Package. R package version 2.0-7.

Olson D.M., Dinerstein E., Wikramanayake E.D., Burgess N.D., Powell G.V.N., Underwood E.C., D’Amico J.A., Itoua I., Strand H.E., Morrison J.C., Loucks C.J., Allnutt T.F., Ricketts T.H., Kura Y., Lamoreux J.F., Wettengel W.W., Hedao P. y Kassem K.R. 2004. Terrestrial ecoregions of the world: A new map of life on earth (ver.2). BioScience 51:933-938.

Pearson R.G., Raxworthy C.J., Nakamura M. y Peterson A.T. 2007. Predicting species distributions from small numbers of occurrence records: a test case using cryptic geckos in Madagascar. Journal of Biogeography 34:102-117.

Peng J., Dan L. y Huang M. 2014. Sensitivity of Global and Re- gional Terrestrial Carbon Storage to the Direct $\mathrm{CO}_{2}$ Effect and Climate Change Based on the CMIP5 Model Intercomparison. PLOS ONE 9:e95282.

Peterson A.T. y Nakazawa Y. 2008. Environmental data sets matter in ecological niche modelling: an example with Solenopsis invicta and Solenopsis richteri. Global Ecology and Biogeography 17:135-144.

Peterson A. T., Papes M., Soberón J. 2008. Rethinking receiver operating characteristic analysis applications in ecological niche modeling. Ecological Modelling 213:63-72.

Peterson A.T., Soberón J., Pearson R.G., Anderson R.P., MartínezMeyer E., Nakamura M. y Araújo M.B. 2011. Ecological Niches and Geographic Distributions. Princeton University Press, Princeton.

Phillips S.J. y Dudik M. 2008. Modeling of species distributions with MaxEnt: new extensions and a comprehensive evaluation. Ecography 31:161-175.

Phillips S.J., Anderson R.P. y Schapire R.E. 2006. Maximum entropy modeling of species geographic distributions. Ecological Modelling 190:231-259.

Pineda-López M.R., Sánchez-Velásquez L.R., Vázquez-Domínguez G. y Rojo-Alboreca A. 2013. The effects of land use change on carbon content in the aerial biomass of an Abies religiosa (Kunth Schltdl. et Cham.) forest in Veracruz, Mexico. Forest Systems 22:82-93.

Parolo G. y Rossi G. 2008. Upward migration of vascular plants following a climate warming trend in the Alps. Basic and Applied Ecology 9:100-107.

Prunier J., Gérardi S., Laroche J., Beaulieu J. y Bousquet J. 2012. Parallel and lineage-specific molecular adaptation to climate in boreal black spruce. Molecular Ecology 21:4270-4286.

Ramírez-Barahona S. y Eguiarte-Fruns L.E. 2013. The role of glacial cycles in promoting genetic diverity in the Neotropics. the case of cloud forests during the Last Glacial Maximum. Ecology and Evolution 3:725-738.

R Core Team. 2014. R: A language and environment for statistical computing. R Foundation for Statistical Computing, Vienna, Austria. <http://www.R-project.org/> (consultado en abril de 2014).

Rushforth K.D. 1989. Two new species of Abies (Pinaceae) from western Mexico. Notes Royal Botanic Garden Edinburgh 46:101-109.

Sáenz-Romero C., Rehfeldt G.E., Duval P. y Lindig-Cisneros R.A. 2012. Abies religiosa habitat prediction in climatic change scenarios and implications for monarch butterfly conservation in Mexico. Forest Ecology and Management 275:98-106.

Sánchez-Velásquez L.R. Plantaciones, ecología y demografía de Abies religiosa y Abies hickelii: Dos especies potenciales para la producción de árboles de navidad en el Cofre de Perote, CONAFOR-2002.-CO1-6163. Fondo Sectorial para la Investigación, el Desarrollo y la Innovación Tecnológica Forestal, Secretaria de Medio Ambiente y Recursos Naturales, México D.F.. Disponible en: <http://www.conafor.gob.mx/biblioteca/ catalogo_fondo_conafor-conacyt-tomo-ii.pdf> (consultado en diciembre de 2012).

Scheldeman X. y van Zonneveld M. 2011. Manual de Capacitación en Análisis Espacial de Diversidad y Distribución de Plantas. Bioversity International, Roma. Disponible en: <http:// www.bioversityinternational.org/e-library/publications/detail/ manual-de-capacitacion-en-analisis-espacial-de-diversidad-ydistribucion-de-plantas/> 
SEMARNAT [Secretaría de Medio Ambiente y Recursos Naturales]. 2007. Anuario Estadístico de la Producción Forestal. Secretaría de Medio Ambiente y Recursos Naturales, México, D.F.

SEMARNAT. 2010. Norma Oficial Mexicana NOM-059-SEMARNAT-2010, Protección ambiental-Especies nativas de México de flora y fauna silvestre-Categorías de riesgo y especificaciones para su inclusión, exclusión o cambio-Lista de especies en riesgo. Diario Oficial de la Federación, 2da Sección, jueves 30 de diciembre de 2010, México D.F.

Semerikova S.A. y Semerikov V.L. 2014. Molecular phylogenetic analysis of the genus Abies (Pinaceae) based on the nucleotide sequence of chloroplast DNA. Russian Journal of Genetics 50:7-19.

Soberón J. y Peterson A.T. 2005. Interpretation of models of fundamental ecological niches and species distributional areas. Biodiversity Informatics 2:1-10.

South A. 2013. rworldmap: A New R package for Mapping Global Data. R package version 1.3-1.

Solorzano, S., M. A. Castillo-Santiago, D. A. Navarrete-Gutiérrez y K. Oyama. 2003. Impacts of the loss of Neotropical highland forests on the species distribution: a case study using resplendent quetzal an endangered bird species. Biological Conservation 114:341-349.

Strandby U., Christensen K.I. y Sørensen M. 2009. A morphometric study of the Abies religiosa-hickelii-guatemalensis complex (Pinaceae) in Guatemala and Mexico. Plant Systematics and Evolution 280:59-76.

Téllez-Valdés O., Chávez-Huerta Y.M., Gómez Tagle-Chávez A. y Gutiérrez-Garduño M.V. 2004. Modelado bioclimático como herramienta para el manejo forestal: estudio de cuatro especies de Pinus. Ciencia Forestal en México 29:61-82.
Telwala Y., Brook B.W., Manish K. y Pandit M.K. 2013. Climateinduced elevational range shifts and increase in plant species richness in a Himalayan biodiversity epicentre. PLOS ONE 8: e57103.

Van Valen L. 1976. Ecological species, multispecies, and oaks. Taxon 25:233-239.

Vázquez-García J.A, Shalisko V., Cuevas-Guzmán R., Muñiz-Castro M.A., Manilla-Blandón M.R. 2014. Abies jaliscana (Pinaceae): A new combination in section Grandis and a key to the species of Abies in western Mexico. Phytotaxa 183:027-036.

Warren D.L., Glor R.E. y Turelli M. 2010. ENMtools: A toolbox for comparative studies of environmental niche models. Ecogoraphy 33:607-611

Wieczorek J., Guo Q. y Hijmas R.J. 2004. The point-radius method for georeferencing locality descriptions and calculating associated uncertainity. International Journal of Geographical Information Science 18:745-767.

Williams C.G. 2009. Conifer Reproductive Biology. Springer., Dordrecht.

Wilson R.J., Gutiérrez D., Gutiérrez J., Martínez D., Agudo R. y Monserrat V.J. 2005. Changes to the elevational limits and extent of species ranges associated with climate change. Ecological Letters 8:1138-1146.

Xiang Q.-P., Wei R., Shao Y.-Z., Yang Z.-Y., Wang X.-Q. y Zhang X.-C. 2015. Phylogenetic relationships, possible ancient hybridization and biogeographic history of Abies (Pinaceae) based on data from nuclear, plastid, and mitochondrial genomes. Molecular Phylogenetics and Evolution 82:1-14

Zeng N., Qian H., Munoz E. y Iacono R. 2004. How strong is carbon cycle-climate feedback under global warming? Geophysical Research Letters 31:L20203.

Recibido: 23 de diciembre de 2014

Aceptado: 28 de abril de 2015 
Apéndice 1. Ecorregiones terrestres del Mundo según la propuesta de Olson et al. 2004, utilizadas para obtener el espacio M para el modelado de nicho ecológico de cada especie.

\begin{tabular}{lll}
\hline OBJECTID & ECO_NAME & G200_REGIO \\
\hline
\end{tabular}

Abies concolor

2248

7744

7603

8376

7735

7737

6795

8094

6925

7204

7299

7309

7434

7437

7780

7781

7783

7786

7537

7551

7589

7643

7645

7648

7656

7659

7705

7730

7749

7759

7762

7769

7771

7795

7861

8068

8147

8157

8183

8188

8351

8457

Abies religiosa

207

855

654

701

754
Klamath-Siskiyou forests

Great Basin montane forests

Northern California coastal forests

Sierra Madre Occidental pine-oak forests

Great Basin montane forests

Great Basin montane forests

North Central Rockies forests

Sonoran desert

California montane chaparral and woodlands

Palouse grasslands

Central and Southern Cascades forests

Eastern Cascades forests

Snake-Columbia shrub steppe

Wyoming Basin shrub steppe

Great Basin montane forests

Great Basin montane forests

Great Basin montane forests

Great Basin montane forests

Great Basin shrub steppe

Western short grasslands

Wasatch and Uinta montane forests

Sierra Nevada forests

Colorado Plateau shrublands

California interior chaparral and woodlands

Great Basin montane forests

California Central Valley grasslands

Great Basin montane forests

Great Basin montane forests

Great Basin montane forests

Great Basin montane forests

Great Basin montane forests

Great Basin montane forests

Great Basin montane forests

Great Basin montane forests

Mojave desert

Arizona Mountains forests

California coastal sage and chaparral

Chihuahuan desert

Arizona Mountains forests

Sierra Madre Occidental pine-oak forests

Sierra Madre Occidental pine-oak forests

Sierra Juarez and San Pedro Martir pine-oak forests
Klamath-Siskiyou coniferous forests

Pacific temperate rainforests

Sierra Madre Oriental and Occidental pine-oak forests

Sonoran-Baja Deserts

California chaparral and woodlands

Sierra Nevada Coniferous Forests

California chaparral and woodlands

California chaparral and woodlands

Chihuahuan-Tehuacán Deserts

Sierra Madre Oriental and Occidental pine-oak forests Sierra Madre Oriental and Occidental pine-oak forests
Trans-Mexican Volcanic Belt pine-oak forests

Sierra Madre del Sur pine-oak forests

Trans-Mexican Volcanic Belt pine-oak forests

Trans-Mexican Volcanic Belt pine-oak forests

Sierra Madre del Sur pine-oak forests
Mesoamerican Pine-Oak Forests

Mesoamerican Pine-Oak Forests

Mesoamerican Pine-Oak Forests

Mesoamerican Pine-Oak Forests

Mesoamerican Pine-Oak Forests 
Apéndice 1. Continuación

\begin{tabular}{lll}
\hline OBJECTID & ECO_NAME & G200_REGIO \\
\hline 765 & Sierra Madre de Oaxaca pine-oak forests & Mesoamerican Pine-Oak Forests \\
807 & Sierra Madre del Sur pine-oak forests & Mesoamerican Pine-Oak Forests \\
9305 & Sierra Madre Occidental pine-oak forests & Sierra Madre Oriental and Occidental pine-oak forests \\
9431 & Sierra Madre Oriental pine-oak forests & Sierra Madre Oriental and Occidental pine-oak forests \\
9444 & Sierra Madre Oriental pine-oak forests & Sierra Madre Oriental and Occidental pine-oak forests \\
9494 & Sierra Madre Oriental pine-oak forests & Sierra Madre Oriental and Occidental pine-oak forests
\end{tabular}

Abies guatemalensis

$927 \quad$ Chiapas montane forests

$148 \quad$ Oaxacan montane forests

163 Central American montane forests

207 Trans-Mexican Volcanic Belt pine-oak forests

$855 \quad$ Sierra Madre del Sur pine-oak forests

271 Central American montane forests

$368 \quad$ Veracruz montane forests

701 Trans-Mexican Volcanic Belt pine-oak forests

$752 \quad$ Petén-Veracruz moist forests

$754 \quad$ Sierra Madre del Sur pine-oak forests

765 Sierra Madre de Oaxaca pine-oak forests

$807 \quad$ Sierra Madre del Sur pine-oak forests

$940 \quad$ Central American pine-oak forests

1008 Sierra Madre de Chiapas moist forests

1073 Central American montane forests

1116 Central American montane forests

$9157 \quad$ Sierra Madre Oriental pine-oak forests

$9431 \quad$ Sierra Madre Oriental pine-oak forests

Abies flinckii

$754 \quad$ Sierra Madre del Sur pine-oak forests

207 Trans-Mexican Volcanic Belt pine-oak forests

Mesoamerican Pine-Oak Forests

Mesoamerican Pine-Oak Forests

Mesoamerican Pine-Oak Forests

Mesoamerican Pine-Oak Forests

Mesoamerican Pine-Oak Forests

Mesoamerican Pine-Oak Forests

Mesoamerican Pine-Oak Forests

Mesoamerican Pine-Oak Forests

Mesoamerican Pine-Oak Forests

Mesoamerican Pine-Oak Forests

Mesoamerican Pine-Oak Forests

Sierra Madre Oriental and Occidental pine-oak forests

Sierra Madre Oriental and Occidental pine-oak forests

Abies hickelii

$148 \quad$ Oaxacan montane forests

$855 \quad$ Sierra Madre del Sur pine-oak forests

$701 \quad$ Trans-Mexican Volcanic Belt pine-oak forests

765 Sierra Madre de Oaxaca pine-oak forests

Abies duranguensis var. durangensis

$8495 \quad$ Sierra Madre Occidental pine-oak forests

Mesoamerican Pine-Oak Forests

Mesoamerican Pine-Oak Forests

Mesoamerican Pine-Oak Forests

Mesoamerican Pine-Oak Forests

Mesoamerican Pine-Oak Forests

Sierra Madre Oriental and Occidental pine-oak forests

Abies durangensis var. coahuilensis

$111 \quad$ Veracruz moist forests

$8748 \quad$ Sierra Madre Oriental pine-oak forests

$9004 \quad$ Sierra Madre Oriental pine-oak forests

$9110 \quad$ Sierra Madre Oriental pine-oak forests

$9113 \quad$ Meseta Central matorral

$9126 \quad$ Sierra Madre Oriental pine-oak forests

Sierra Madre Oriental and Occidental pine-oak forests Sierra Madre Oriental and Occidental pine-oak forests Sierra Madre Oriental and Occidental pine-oak forests Chihuahuan-Tehuacán Deserts

Sierra Madre Oriental and Occidental pine-oak forests

Abies vejarii

9110

Sierra Madre Oriental pine-oak forests

Sierra Madre Oriental and Occidental pine-oak forests 
Apéndice 2. Prueba no paramétrica de comparaciones múltiples de Gao.

Prueba no paramétrica de comparaciones múltiples de Gao entre pares de especies y el componente 1 del ACP. Solamente se muestran las comparaciones sin diferencias significativas (alpha $=0.05$ ).

\begin{tabular}{|c|c|c|c|c|}
\hline \multirow[t]{2}{*}{ Comparaciones } & \multicolumn{4}{|c|}{ CP1 } \\
\hline & Estadístico & g.l. & p.BONF & p.HOLM \\
\hline $\begin{array}{l}\text { Abies durangensis var. coahulensis - A. durangensis } \\
\text { var. durangensis }\end{array}$ & 1.3485 & 18.7365 & 1 & 1 \\
\hline A. durangensis var. coahulensis - A. vejarii & 3.2787 & 14.6592 & 0.2865 & 0.1146 \\
\hline A. flinckii s.l.- A. durangensis var. coahuilensis & 2.6914 & 16.726 & 0.8584 & 0.2965 \\
\hline A. flinckii s.l.- A. flinckii & 1.8324 & 47.9472 & 1 & 1 \\
\hline A. flinckii s.I.- A. guatemalensis & 0.5587 & 76.2378 & 1 & 1 \\
\hline A. flinckii - A.durangensis var. coahulensis & 1.6088 & 18.7864 & 1 & 1 \\
\hline A. flinckii - A. religiosa & 2.9833 & 47.0582 & 0.248 & 0.1037 \\
\hline A. guatemalensis - A. durangesis var. coahulensis & 2.0861 & 24.251 & 1 & 0.7624 \\
\hline A. guatemalensis - A. flinckii & 0.8773 & 69.4082 & 1 & 1 \\
\hline A. hickelii - A. durangensis var. coahulensis & 2.7254 & 20.2517 & 0.7116 & 0.2588 \\
\hline A. hickelii - A. flinckii & 1.8531 & 57.3776 & 1 & 1 \\
\hline A. hickelii - A. flinckii s.l. & 0.3037 & 68.5091 & 1 & 1 \\
\hline A. hickelii - A. guatemalensis & 0.7398 & 84.5798 & 1 & 1 \\
\hline A. jaliscana - A. flinckii s.l. & 2.5165 & 43.4324 & 0.8593 & 0.2965 \\
\hline A. jaliscana - A. guatemalensis & 2.4021 & 64.4363 & 1 & 0.3263 \\
\hline A. jaliscana - A. hickelii & 1.8304 & 52.4764 & 1 & 1 \\
\hline A. religiosa - A. durangensis var. durangensis & 2.7389 & 70.2754 & 0.4293 & 0.1639 \\
\hline A. religiosa - A.durangensis var. coahulensis & 0.0631 & 15.9778 & 1 & 1 \\
\hline
\end{tabular}

p.Bonf $=$ Valores de probabilidad ajustados de Bonferroni

p. Holm = Valores ajustados de probabilidad de Holm.

Prueba no paramétrica de comparaciones múltiples de Gao entre pares de especies y el componente 2 del ACP. Solamente se muestran las comparaciones sin diferencias significativas (alpha $=0.05$ ).

\begin{tabular}{|c|c|c|c|c|}
\hline \multirow[t]{2}{*}{ Comparaciones } & \multicolumn{4}{|c|}{ CP2 } \\
\hline & Estadístico & g.l. & p.BONF & p.HOLM \\
\hline Abies concolor - A.durangensis var. coahulensis & 1.7324 & 15.3291 & 1 & 1 \\
\hline A. concolor - A. flinckii & 2.8945 & 41.9042 & 0.3303 & 0.1622 \\
\hline $\begin{array}{l}\text { A. durangensis var. durangensis - A. durangensis } \\
\text { var. coahulensis }\end{array}$ & 0.3135 & 18.2523 & 1 & 1 \\
\hline A. durangensis var. durangensis - A. flinckii & 0.2013 & 51.5085 & 1 & 1 \\
\hline A. durangensis var. durangensis - A. flinckii s.l. & 1.6137 & 64.5142 & 1 & 1 \\
\hline A. durangensis var. durangensis - A. jaliscana & 3.21 & 48.3017 & 0.1299 & 0.0779 \\
\hline A. durangensis var. coahulensis - A.flinckii s.l. & 0.5218 & 16.023 & 1 & 1 \\
\hline A. durangensis var. coahulensis - A. jaliscana & 1.4264 & 16.7833 & 1 & 1 \\
\hline A. flinckii s.l. - A. jaliscana & 1.9224 & 42.6356 & 1 & 1 \\
\hline A. flinckii - A. durangensis var. coahulensis & 0.1937 & 18.7124 & 1 & 1 \\
\hline A. flinckii - A.flinckii s.l. & 1.3325 & 44.2561 & 1 & 1 \\
\hline A. flinckii - A. jaliscana & 2.8938 & 38.8191 & 0.3417 & 0.1622 \\
\hline A. guatemalensis - A. durangensis var. durangensis & 0.4955 & 80.7365 & 1 & 1 \\
\hline A. guatemalensis - A. durangensis var. coahulensis & 0.6016 & 20.0041 & 1 & 1 \\
\hline A. guatemalensis - A. flinckii & 0.6719 & 63.5042 & 1 & 1 \\
\hline
\end{tabular}


Apéndice 2. Continuación

\begin{tabular}{|c|c|c|c|c|}
\hline \multirow[t]{2}{*}{ Comparaciones } & \multicolumn{4}{|c|}{ CP2 } \\
\hline & Estadístico & g.l. & p.BONF & p.HOLM \\
\hline A. guatemalensis - A. flinckii s.l. & 2.0289 & 81.1116 & 1 & 1 \\
\hline A. hickelii - A.durangensis var. coahulensis & 2.4996 & 19.6249 & 1 & 0.5145 \\
\hline A. hickelii - A. guatemalensis & 3.0103 & 83.9783 & 0.1895 & 0.11 \\
\hline A. religiosa - A. durangensis var. coahulensis & 3.4838 & 14.6478 & 0.1891 & 0.11 \\
\hline A. religiosa - A. hickelii & 1.4628 & 57.075 & 1 & 1 \\
\hline A. vejarii - A. durangensis var. coahulensis & 3.2615 & 21.5906 & 0.2 & 0.11 \\
\hline A. vejarii - A. hickelii & 1.2886 & 53.6592 & 1 & 1 \\
\hline A. vejarii - A. religiosa & 0.2647 & 33.7075 & 1 & 1 \\
\hline
\end{tabular}

Prueba no paramétrica de comparaciones múltiples de Gao entre pares de especies y el componente 3 del ACP. Solamente se muestran las comparaciones sin diferencias significativas (alpha $=0.05$ ).

\begin{tabular}{|c|c|c|c|c|}
\hline \multirow[t]{2}{*}{ Comparaciones } & \multicolumn{4}{|c|}{ CP3 } \\
\hline & Estadístico & g.l. & p.BONF & p.HOLM \\
\hline A. concolor - A. durangensis var. coahulensis & 3.6907 & 16.1505 & 0.1075 & 0.0645 \\
\hline $\begin{array}{l}\text { A. durangensis var. durangensis - A. durangensis } \\
\text { var. coahulensis }\end{array}$ & 3.0226 & 16.1498 & 0.4413 & 0.2166 \\
\hline A. durangensis var. durangensis - A. guatemalensis & 3.0329 & 63.3213 & 0.193 & 0.1053 \\
\hline A. durangensis var. durangensis -A. hickelii & 1.187 & 49.7901 & 1 & 1 \\
\hline A. durangensis var. durangensis - A. jaliscana & 3.2744 & 40.3589 & 0.1198 & 0.0697 \\
\hline A. durangensis var. coahulensis - A. flinckii & 1.9966 & 18.2106 & 1 & 1 \\
\hline A. durangensis var. coahulensis - A. flinckii s.l. & 0.5317 & 17.7076 & 1 & 1 \\
\hline A. durangensis var. coahulensis - A. religiosa & 2.9817 & 16.8963 & 0.4629 & 0.2188 \\
\hline A. flinckii s.I. - A.flinckii & 2.6454 & 54.4595 & 0.5853 & 0.2554 \\
\hline A. flinckii - A. religiosa & 1.7207 & 58.6953 & 1 & 1 \\
\hline A. guatemalensis - A. durangensis var. coahulensis & 0.3781 & 32.5583 & 1 & 1 \\
\hline A. guatemalensis - A.flinckii & 2.8584 & 67.5103 & 0.3112 & 0.1584 \\
\hline A. guatemalensis - A. flinckii s.l. & 1.155 & 70.1072 & 1 & 1 \\
\hline A. hickelii - A. durangensis var. coahulensis & 1.6789 & 30.7958 & 1 & 1 \\
\hline A.hickelii - A. flinckii s.l. & 3.0133 & 55.6668 & 0.2136 & 0.1126 \\
\hline A. hickelii - A. guatemalensis & 1.4463 & 84.0131 & 1 & 1 \\
\hline A. hickelii - A. jaliscana & 0.7532 & 50.6117 & 1 & 1 \\
\hline A. hickelii - A. vejarii & 1.4738 & 49.0657 & 1 & 1 \\
\hline A. jaliscana - A. durangensis var. coahulensis & 1.3759 & 17.0776 & 1 & 1 \\
\hline A. jaliscana - A. guatemalensis & 1.0834 & 63.2229 & 1 & 1 \\
\hline A. jaliscana - A. vejarii & 1.173 & 37.1516 & 1 & 1 \\
\hline A. vejarii - A. durangensis var. coahulensis & 0.8196 & 16.0957 & 1 & 1 \\
\hline A. vejarii - A. flinckii s.l. & 2.6796 & 63.7801 & 0.5152 & 0.2342 \\
\hline A. vejarii - A. guatemalensis & 0.4221 & 62.2643 & 1 & 1 \\
\hline
\end{tabular}


Apéndice 3. Porcentaje del área de los nichos ecológicos potenciales de Abies en México que están dentro de un Área Natural Protegida (ANP).

\begin{tabular}{lccccc}
\hline Especie & $\begin{array}{c}\text { \% Total } \\
\text { en México }\end{array}$ & $\begin{array}{c}\text { \% Del total en } \\
\text { México dentro } \\
\text { de una ANP }\end{array}$ & \multicolumn{2}{c}{$\begin{array}{c}\text { \% Del total en México por nivel de } \\
\text { idoneidad del hábitat dentro } \\
\text { de una ANP }\end{array}$} \\
\cline { 5 - 6 } & & $18.62(0)$ & 17.07 & 9.51 & 63.87 \\
\hline Abies concolor & 3.10 & $31.57(41.67)$ & 20.59 & 47.00 & 60.90 \\
Abies religiosa & 100 & $13.10(8.16)$ & 13.15 & 16.37 & 8.90 \\
Abies guatemalensis & 68.56 & 13.35 & 10.18 & 17.51 & 22.92 \\
Abies guatemalensis s.l. & 78.60 & $8.50(26.09)$ & 4.61 & 9.08 & 33.20 \\
Abies flinckii & 100 & $18.55(19.51)$ & 18.47 & 15.44 & 31.94 \\
Abies flinckii s.l. & 100 & $19.47(7.89)$ & 16.01 & 27.23 & 24.30 \\
Abies hickelii & 100 & $15.70(20.59)$ & 16.40 & 13.41 & 17.45 \\
Abies durangensis var. durangensis & 100 & $24.62(78.57)$ & 18.26 & 44.78 & 63.65 \\
Abies durangensis var. coahuilensis & 100 & $42.28(56)$ & 38.50 & 50 & 76.16 \\
Abies vejarii & 100 & $15.98(11.11)$ & 20.86 & 1.96 & 1.96 \\
Abies jaliscana & 100 & & & Medio \\
\hline
\end{tabular}

Nota 1. Las áreas fueron calculadas a partir del umbral de mínima presencia de entrenamiento de los puntos de entrenamiento de cada modelo.

Nota 2. Los porcentajes dentro de una ANP por nivel de idoneidad del hábitat no suman $100 \%$, ya que las áreas protegidas no incluyen de manera proporcional los distintos niveles de idoneidad.

Nota 3. Entre paréntesis se muestra el porcentaje de los puntos de colecta utilizados para realizar los modelos que caen dentro de una ANP. 